\title{
A Non-stop identity complex (NIC) supervises enterocyte identity and protects from pre-
}

\section{mature aging}

Neta-Erez ${ }^{1}$, Lena Israitel ${ }^{1}$, Eliya Bitman-Lotan ${ }^{1}$, Wing Hing Wong ${ }^{2}$ Gal Raz ${ }^{1}$, Salwa Danial ${ }^{1}$, Na'ama Flint Brodsly ${ }^{1}$, Elena Belova ${ }^{3}$ Oksana Maksimenko $^{3}$, Pavel Georgiev ${ }^{3}$, Todd Druley ${ }^{2}$, Ryan Mohan $^{4}$, and Amir Orian ${ }^{\S 1}$

${ }^{1}$ Rappaport Research Institute and Faculty of Medicine, Technion-Israel Institute of Technology, Haifa, 3109601 Israel.

${ }^{2}$ Division of Pediatric Hematology and Oncology, Washington University, Saint-Louis MO 63110, USA.

${ }^{3}$ Department of the Control of Genetic Processes, Institute of Gene Biology Russian Academy of Sciences, Moscow, 119334 Russia

${ }^{4}$ Department of Genetics, Developmental \& Evolutionary Biology, School of Biological and Chemical Sciences University of Missouri, Kansas City, MO 64110-2239 USA

Running title: Non-stop regulates enterocyte identity.

Keywords: Aging, Cell identity, Drosophila, Gut, Gene regulation, Ubiquitin, USP22/ Non-stop. $\S$ Lead Contact and Author for correspondence:

$$
\text { Amir Orian } \mathrm{MD} / \mathrm{PhD}
$$

email: mdoryan@tx.technion.ac.il

Tel: $972-4-829-5448 / 9$

Fax: 972-4-852-394 


\section{Summary: 238w}

A hallmark of aging is the inability of differentiated cells to maintain their identity. In the aged Drosophila midgut differentiated enterocytes (ECs) lose their identity, and the integrity of the midgut tissue and its homeostasis are impaired. To discover regulators of EC identity relevant to aging we performed an RNAi screen targeting 453 ubiquitin-related genes in fully differentiated ECs. Seventeen genes were identified, including the de-ubiquitinase Non-stop (Not/dUSP22; CG4166). Acute loss of Non-stop in young ECs phenotypically resembled aged ECs. Lineage tracing experiments established that Non-stop-deficient young ECs as well as wild-type aged ECs are no longer differentiated. Aging or acute loss of Non-stop also resulted in progenitor cell hyperproliferation and mis-differentiation, loss of gut integrity, and reduced organismal survival. Proteomic analysis unveiled that Non-stop maintains identity as part of a Non-stop identity complex (NIC) that contains E(y)2, Sgf11, Cp190, (Mod) mdg4, and Nup98. Transcriptionally, Non-stop ensured chromatin accessibility at EC genes, maintained an ECspecific gene expression signature, and silenced non-EC-relevant transcriptional programs. Within the NIC, Non-stop was required for stabilizing of NIC subunits. Upon aging, the levels of Non-stop and NIC subunits declined, and the large-scale organization of the nucleus was distorted. Maintaining youthful levels of Non-stop in wildtype aged ECs safeguarded the protein level of NIC subunits, restored the large-scale organization of the differentiated nucleus, and suppressed aging phenotypes and tissue integrity. Thus, the isopeptidase Non-stop, and NIC, supervise EC identity and protects from premature aging. 


\section{Introduction:}

Differentiated cell states are actively established and maintained through action of "identity supervisors" (Holmberg \& Perlmann, 2012; Natolli, 2014). Identity supervisors control expression of genes that enable differentiated cells to respond to environmental cues and perform required physiological tasks. Concomitantly, they ensure silencing/repression of previous fate and non-relevant gene programs and reduce transcriptional noise. Inability to safeguard differentiated cell identity is a hallmark of aging and results in diseases such as neurodegeneration, diabetes, and cancer (Bensellam et al. 2018; Hudish, et al. 2019; Conway et al 2015; Hnisz et al. 2013; Deneris \& Hobert 2014). In many cases, transcription factors (TFs) together with chromatin regulators and architectural/scaffold proteins establish and maintain large-scale chromatin and nuclear organization that is unique to the differentiated state of the cell (Blau \& Baltimore 1991; Booth \& Brune 2016; Naetar, et al 2017; Bitman-Lotan \& Orian 2018).

In adult Drosophila midgut epithelia, the transcription factor Hey (Hairy/E(spl)-related with YRPW motif), together with Drosophila nuclear type A lamin, Lamin C (LamC), co-supervise identity of fully differentiated enterocytes (ECs) (Monastirioti et al. 2010; Gruenbaum and Foisner R. (2015)Flint-Brodsly et al. 2019). Highly similar to vertebrate gut, Drosophila midgut epithelia intestinal stem cells (ISC) either self-renew or differentiate into progenitor cells that mature into enteroendocrine cells (EEs) or give rise to enteroblast progenitors. Enteroblasts (EB) mature into fully polyploid differentiated enterocytes (ECs) that carry out many critical physiological tasks of the intestine (Figure 1A; Jiang, \& Edgar 2012; Lemaitre \& MiguelAliaga 2013; Buchon et al. 2013; Hung et al. 2020). Aging affects the entire midgut, and is associated with loss of EC identity, mis-differentiation of progenitors, pathological activation of the immune system, and loss of the physiological properties of the gut and its integrity. It 
also results in loss of intestinal compartmentalization, and microbiota-dysbiosis, all leading to reduced lifespan (Biteau et al. 2010; Rera et al 2012; Bonnay et al. 2013; Ferrandon, 2013; Chen et al. 2014; Li, et al. 2020; Rodriguez-Fernandezet al 2020; Jasper H. 2020). During aging, the protein levels of identity supervisors such as Hey and LamC decline, resulting in inability to maintain EC-gene programs and ectopic expression of previous- and non-relevant gene programs (Neves et al 2015; Takeda et al 2018; Flint-Brodsly et al. 2019). Indeed, continuous expression of Hey in aged ECs restores and protects EC identity, gut integrity, and tissue homeostasis (Flint-Brodsly et al. 2019).

Regulation of EC identity requires signaling to the nucleus to communicate physiological changes in the gut environment. An important dynamic signaling mechanism involves changes in post-transcriptional modifications which may propagate, amplify, or conduct signals, ultimately leading to differential gene regulation. One type of posttranslational modification is the covalent attachment of ubiquitin or ubiquitin-like (Ub/UbL) molecules, that affect protein stability, function, localization, as well as modulate chromatin structure (Heideker, Wertz; 2015; Swatek\& Komander, (2016); Cappadocia, \& Lima, 2018; Song \& Luo, 2019; Yao et al. 2020). Recent works suggest an intimate links between ubiquitin proteostasis and aging (Kevei, \& Hoppe, 2014; Vilchez et al. 2014; Hohfled \& Hoppe, 2018; Enam et al 2018; Chua \& Signer, 2020). Therefore, we performed an RNAi screen to search for Ub/UbL-related genes within ECs that supervise identity. Screening 548 genes, 17 were identified whose conditional elimination in fully differentiated ECs resulted in loss of EC identity. Further analysis revealed one of them, the deubiquitinating isopeptidase (DUB) Non-stop (Non-stop/dUSP22) is a key EC identity supervisor. Purification and proteomic analysis identified Non-stop as part of a CP190/Nup98/Sgf11/e(y)2/mdg4 protein complex, termed Non-stop identity complex (NIC), that is essential for maintenance of EC identity. In part, Non-stop protects NIC proteins from age-dependent decline, safeguarding the EC-gene expression signature, as well as large-scale 
nuclear organization in these cells, preventing premature aging. Over lifespan, Non-stop protein levels in ECs declined, leading to loss of NIC subunits. This decline is associated with loss of gut identity and physiology at the cellular and tissue levels and maintaining youthful levels of Non-stop prevented loss of the NIC and prevented aging of the gut.

\section{Results:}

A transgenic RNAi screen identified $\mathrm{Ub} / \mathrm{UbL}-$ related EC identity regulators. To identify EC identity supervisors, a collection of RNAi transgenic flies targeting 453 evolutionarily conserved Ub/UbL-related genes were screened (Table S1; List of Ubiquitin-Related Genes according to DRSC - http://www.flyrnai.org/DRSC-SUB.html) .Genes were knocked-down in fully differentiated ECs of 2-4 day old adult Drosophila females using UAS-RNAi lines and EC-specific conditional driver $M y o I A-G a 14 / G a 180^{\text {ts }}$ coupled system (termed MyoIA ${ }^{\text {ts. }}$, see methods for specific lines used; Salmeron et al., 1990; Brand and Perrimon, 1993; Flint Brodsly et al. 2019). Conditional RNAi was achieved by shifting flies from 25 to $29^{\circ} \mathrm{C}$ for 48 hours, after which guts were dissected and analyzed. Immunofluorescence was used to score loss of proteins which are hallmarks of EC identity (Figure 1A, B). Among the changes upon loss of EC identity is the as ectopic expression of the ISC marker Delta on the surface of ECs-like polyploid cells. This change may be also accompanied with a decline in the expression of a GFP signal expressed only in fully differentiated ECs (derived from the MyoIA ${ }^{\text {ts }}$ Gal4, UASGFP transgene). Knockdown of seventeen genes resulted in loss of EC identity and the appearance of EC-like polyploid cells (PPCs). We also determined whether these genes are required for maintaining identity of enteroendocrine cells (EE's), or progenitor cells, using the Prospero $>\mathrm{Gal} 4 / \mathrm{Gal} 80^{\text {ts }}$, or Esg $>\mathrm{Gal} 4 / \mathrm{Gal} 0^{\text {ts }}$ that activates the UAS-RNAi in these cells, respectively (Figure 1, Supplemental Table S1). 
Non-stop supervises EC identity. Among the genes identified were E3 ubiquitin ligases, E2 enzymes, SUMO-related enzymes and ubiquitin-specific peptidases (DUB/USPs). We also identified few nuclear proteins harboring PHD domain that serve as binding to methylated histones, but may confer ubiquitin ligase activity present in established ubiquitin ligases (Examples are shown in Figure1, Supplemental Figure 1A-H,). The entire results of the screen are detailed in Figure 1, Supplemental Table S1 section of the table and validated positive hits under secondary screen). The DUB Non-stop (Not, dUSP22, CG4166) was identified as a bonafide EC identity supervisor. RNAi mediated knockdown of Non-stop in ECs using three independent UAS-RNAi lines resulted in the inability of ECs to maintain MyoIA>UAS-GFP signal followed by ectopic expression of the ISC marker Delta on the surface of EC-like polyploid cells in both females and males (Figures 1C, 1D, quantitated in $1 \mathrm{G}$ and Figure 1 Source-data; Figure 1 Figure supplemental 1I-K).

Originally, Non-stop was discovered as a ubiquitin protease essential for axonal guidance in the visual system (Martin et al 1995). Non-stop is highly conserved from yeast to humans (Ubp8 and USP22 respectively; Mohan et al. 2014b), and its activity is required for deubiquitinating monoubiquitinated histone $\mathrm{H} 2 \mathrm{~B}$ (H2Bub) and activating gene expression (Weake et al. 2008; Mohan et al. 2014; Morgan et al 2016).

Immunofluorescence revealed that Non-stop is expressed in all gut cells (Figure 1 Supplemental Figure 2A-D). Non-stop is the major H2Bub deubiquitinase in Drosophila, therefore functional loss of Non-stop should lead to a many-fold increase in H2Bub levels (Weake et al. 2008; Zhang et al 2008; Morgan et al. 2016; Mohan et al, 2014; Li et al. 2017). Analysis showed that ECs lacking Non-stop exhibited more H2Bub, and accordingly protein extracts derived from these midguts were characterized by an over 6-fold increase in H2Bub compared to control knockdown midguts (Figure 1 Supp. Figure 2F-H). However, reduction of Non-stop in EEs did not impact EE or EC identity or number, or Delta expression, indicating that Non-stop function in maintaining gut identity was specifically localized in ECs (Figure 1 Supp. Figure 2I-L). 
Non-stop knockdown in ECs resulted in an increase in Prospero positive cells (likely EEs) (Figure 1E, F, and quantified in 1H, Figure 1 Source-data). Non-stop elimination also affected the entire midgut tissue; resulting in ectopic activation of the Notch pathway, as well as the stem-cell enhancer M5-4 esg::LacZ in polyploid cells, indicating that these cells were losing differentiated state (Figure 1I-L, 1Q, and Figure 1 source data). Loss of Non-stop also resulted in increased phospho-Histone $\mathrm{H} 3$ which is indicative of mitotic activity in small cells, likely progenitors (Figure 1M, N).

At the tissue level, knockdown of Non-stop in ECs reduced epithelial integrity as evidenced by leaking of blue colored food outside the gut, and reduced overall survival (Figure 1O, P and 1R respectively; Figure 1 source data).

We evaluated the identity and fate of young ECs conditionally lacking Non-stop, as well as aged wildtype ECs, as well as the cellular composition of the gut under these conditions. Towards this end we used the lineage tracing system G-TRACE. G-TRACE is a dual-color GAL4-dependent system, that enables tracing fully differentiated non-dividing cells (Figure 2A; Evans et al. 2009; Flint-Brodsly et al. 2019). In brief, the MyoIA-Gal4/Gal80 $0^{\text {ts }}$ directs the expression of the color system only to fully differentiated ECs. Expression of the Gal4 is dependent on the EC-specific MyoIA promoter that induces expression of a UAS-RFP (red). Concomitantly, this Gal4 activity induces the expression of a Flp-recombinase resulting in a recombination event that drives permanent expression of a GFP regardless of the differentiation state of the cell. Therefore, wildtype young ECs express both RFP and GFP, and are the only population of polyploid cells (PPCs), observed in control midgut tissue (Figure 2B). In contrast, in guts where Non-stop was knocked-down in ECs, other populations of fluorescently colored PPC's were observed. These include PPCs that express only GFP, termed PPC** (PPC GFP+ RFP; Figure $2 \mathrm{C}$, and quantitated in $2 \mathrm{H}$; Figure 2 source data). Unlike control cells, PPC** did not express EC-related transcription factors such Odd-skipped (Figure 2D-E). They also exhibited reduced expression of the differentiated lamin, LamC, (Figure 2F, G). By the nature of the G- 
TRACE system, we concluded that these PPC** are likely ECs that were no longer fully differentiated, failing to maintain EC identity. In accordance, PPCs that did not express EC key transcription factors such as Pdm1 and caudal were also observed (Figure 2 Figure Supplemental 1A-D). In addition, guts where Non-stop was targeted in ECs were populated with PPCs lacking expression of either RFP or GFP (termed PPC*), and are likely misdifferentiated progenitors that failed to activate the myo-promoter and the entire RFP/GFP marking system (Figure 2H, Figure 2 Source data).

The phenotypes observed upon acute loss of Non-stop are highly similar to the ones observed in aged midguts (Figure 2I-P; Figure 2 Supplemental Figure 2, 3). G-TRACE analysis of aged ECs established that the aged midgut (5 weeks old) are populated with ECs that are no longer differentiated $\left(\mathrm{PPC}^{* *}\right)$, as well as mis-differentiated progenitors $\left(\mathrm{PPC}^{*}\right)$. These $\mathrm{PPC} * *$ nolonger expressed the differentiated LamC, or the transcription factors Pdm1 and Odd-skipped and ectopically expressed the stem cell marker Delta (Figure 2I-O, quantitated in Figure 2P, and Figure 2 supplemental Source data; and Figure 2 Figure Supplemental 3A-D).

As in the case of young ECs lacking Non-stop, aging ECs ectopically expressed the stem cell enhancer M5-4 (Figure 2 Figure Supplemental 2 A, B, I, J). They also exhibited reduced expression of the differentiated Lamin LamC (Figure 2 Figure Supplemental 2C, D, K, L) and ectopic expression of the stem cell-related Lamin, LamDm0 as well as its binding partner Otefin (Ote) in PPC (Figure 2 Figure Supplemental 2E, F, M, N, G, H). At the tissue level, aged ECs also exhibited disorganized distribution of EC-related adhesion molecule Disc large, (Dlg), and reduced expression of MESH and snakeskin (SSK). They ectopically express Armadillo (Drosophila $\beta$-catenin), which s expressed on the surface of progenitors in young midguts, all resulting in loss of gut integrity (Figure 2 Figure Supplemental 3E-L; Figure 9O, P). Thus, acute loss of Non-stop in young EC or age-related declines in aged ECs results in EC cells that lose differentiation status and/or mis-differentiate. 
A Non-stop identity complex (NIC) supervises EC identity: Non-stop is the catalytic subunit of a DUB module containing Sgf11, E(y)2 and in some cases Ataxin7 that is part of the SAGA chromatin remodeling complex (Morgan and Wolberger 2017, Mohan 2014, Weake 2008). We therefore tested whether the SAGA complex is required for maintaining EC identity. ECspecific RNAi-mediated reduction of Ataxin7 (part of the DUB module), or GCN5 (the histone acetyl transferase of the SAGA complex) did not result in loss of EC identity (Figure 3, Sup. Figure 1 and not shown). We concluded that the EC identity-related function(s) of Non-stop are independent of SAGA.

Therefore, we biochemically searched for Non-stop-associated proteins that potentially together maintain EC identity. Toward this end, we generated a Drosophila S2 cell line stably expressing epitope-tagged Non-stop-2xFLAG-2xHA (Non-stop-FH) under the control of a copper-sulfate-responsive metallothionein promoter. Protein complexes that contained Nonstop were affinity purified using sequential capture of the epitope tags, FLAG, then HA. These complexes were subsequently resolved according to size, using gel filtration chromatography (Figure 3A-C). We used a ubiquitin-AMC de-ubiquitinase activity assay to track enzymatically active Non-stop in the purified fractions (Figure 3B). We found three major peaks of deubiquitinase activity. The major activity peak resolved at about $1.8 \mathrm{MDa}$, together with components of SAGA complex (Group 1). A second peak was resolved centering approximately around $670 \mathrm{kDa}$ (Group 2). A third peak, with the lowest total activity, was detected centering around $75 \mathrm{kDa}$. The three fractions comprising the center of each peak were combined and constituent proteins identified by mass spectrometry (MudPIT) (Washburn et al., 2001). Group 2 contained e(y)2 and Sgf11but no other SAGA subunits. Remarkably, it also contained members of a known boundary complex that includes Cp190, Nup98, Mod (mdg4) and is known to be part of nuclear complex regulating enhancer-promotor interactions and affecting transcriptional memory (Pascual-Garcia et al 2017). We also noted that Histones H2A and $\mathrm{H} 2 \mathrm{~B}$ were also detected in both groups 1 and 2, showing the DUB module was co-purifying 
with known substrates of Non-stop and indicating the DUB was purifying in a physiologically native state (Figure 3C; Zhao et al., 2008).

We mapped the interaction of Non-stop with members of the complex using in vitro binding assays and yeast two-hybrid system (Y2H, Figure 3D-F). In vitro binding, using S2 cell-derived extract expressing HA-Non-stop and His-tagged proteins, established that Non-stop interacted with its known interaction partner e(y)2 as well as with the C-terminal portion of Cp190 (amino acids (a. a.) 468-1096), but minimally with the N-terminal portion of Cp190 (a.a. 1-524). Additionally, in the Y2H system, Non-stop interacted with full length Cp190. Y2H mapped this interaction to the second and third zinc fingers of Cp190 but not the first or fourth (Figure 3F). Non-stop did not interact with either Nup98 or Mod (mdg4) in similar binding assays (not shown).

We termed this complex NIC (Non-stop identity complex) and hypothesized that if the NIC supervises EC identity, RNAi-mediated elimination of each of its subunits will result in loss of EC identity similar to the loss of Non-stop. Indeed, EC-specific knockdown of all NIC subunits except Sgf11 resulted in loss of identity and inability to maintain expression of the EC gene LamC (Figure 4A-F, Figure 3 Figure Supplemental 1). It also resulted in ectopic expression of Delta (Figure 4G-L). In contrast, loss of $\mathrm{Su}(\mathrm{Hw})$, an insulator protein that binds to Mod (mdg4) but was not identified as a Non-stop binding partner, did not result in any detectable phenotype (Figure 3, Figure supplemental 1E).

Non-stop supervises EC-gene signature and regulates chromatin accessibility. Non-stop is well known to regulate gene expression (Mohan et al. 2014, Li et al. 2017). To elucidate Non-stop-dependent expression signatures, we determined the changes in transcriptional expression using RNA-Seq and its effect(s) on chromatin accessibility by ATAC-seq analyses 
(Figure 5). We determined the changes in transcription signatures of whole guts upon elimination of Not in ECs using UAS-Not-RNAi and the EC-specific MyoIA-Gal4 ${ }^{\text {ts }}$ We identified 863 genes with downregulated mRNA expression upon loss of Not in ECs (Table S1 and Figure 5 Figure Supplemental 1D). Of these, 38\% (398/1039) were previously identified as EC-related genes (Figure 5A; Korzelius 2014). Metascape analysis unveiled that these shared targets consist of core EC pathways that execute many of the physiological tasks of the gut (Figure 5B; Figure 5 Supplemental Table 1, 2).

We previously identified genes that required Hey for their expression in ECs, and 76\% (174/228) of Hey-dependent genes also required Not for expression (Figure 5C).

Moreover, the expression of EC-specific genes was repressed by ectopic expression of the ISCrelated lamin, LamDm0, in ECs (Flint-Brodsly 2019). Fifty percent (188/372) of genes that are repressed by expression of LamDm0 in ECs also required Not for their expression, and 46 of these genes were regulated also with Hey (Figure 5D, Figure 5 Figure supplemental 1A-B and see discussion).

In parallel, we examined whether expression of EC-genes involves Non-stop-dependent regulation of chromatin accessibility using ATAC-seq. We identified 214 loci that exhibited reduced chromatin accessibility ("closed"). Of these, 75\%(162/214) were located in the range of $0-10 \mathrm{~Kb}$ vicinity of genes that exhibited reduced expression (Figure $5 \mathrm{~A}$; Figure 5 Supplemental Figure 3A, and Supplemental Table S3, S4). GO analysis of these "closed" regions suggested that they belong to genes that maintain the physiological properties of enterocytes (Figure 5E). Alignment of the "closed" chromatin regions showed that they cluster to discrete gene regions (Figure 5F; for EC-related down-regulated genes, and Figure 5, Supplemental Figure 2A for all closed sites, Supplemental Table S5). As shown in Figure 5F, a one cluster was located to the 5' UTR, a second cluster was at the transcriptional start site (TSS), a third was spanning the coding region, and a fourth was located at the 3'-UTR. MEME 
analysis revealed that they are statistically significantly enriched in DNA motifs that are known binding sequences of TFs (Figure 5F, Figure 5 Supplemental Figure 2B).

In addition, 565 genes showed upregulation of mRNA expression upon loss of Non-stop, and are related to progenitor fate, cell cycle, and DNA repair (Figure 5, Supplemental 1C). In contrast to numerous closed regions only a small number $(\sim 16)$ regions exhibited increased accessibility upon loss of Non-stop in ECs interestingly many of these genes code of long noncoding RNA (Figure 5 Figure supplemental 2C). Thus, supporting the notion that Non-stop acts primarily to maintain chromatin accessibility in the vicinity of its targets.

We hypothesized that the ectopic expression of these genes may be, at least partially, due to changes in nuclear organization in ECs. In this regard, among the genes that require Nonstop/NIC at the protein level is LamC (Figure 4A-F). LamC is the dominant lamin in ECs that silences the expression of stem cell and non-relevant gene programs in ECs (Flint-Brodsly 2019). For example, PCNA is not expressed in control ECs, but is ectopically expressed in $\mathrm{PPC}^{* *}$ (ECs that are no longer differentiated; $\mathrm{PPC}^{\mathrm{GFP}+\mathrm{RFP}-}$ ). This ectopic expression was prevented by co-expression of LamC in ECs where Non-stop was eliminated (Figure 5, Supplemental Figure 1E-G). Moreover, loss of Non-stop in ECs also resulted in a significant decrease in the linker histone $\mathrm{H} 1$ that is associated with compacting chromatin and gene silencing (Fyodorov et al. 2018). H1 protein levels were reduced in the nuclear periphery of ECs lacking Non-stop (Figure 5G, H), in gut extracts derived from flies where Non-stop was targeted in ECs, as well as upon knockdown of Non-stop in S2R cells (Figure 5I, J respectively). Thus, the ectopic expression of non-EC programs may be due loss of LamC and H1 proteins and subsequently heterochromatin impairment and dependent silencing. However, since we isolated mRNA from the entire midgut, the source of these upregulated mRNAs may also be from mis-differentiated stem cells ( $\left.\mathrm{PPC}^{*}\right)$, as well as from the increase in rapidly dividing progenitor cells. 
Comparison of Non-stop RNA-seq data with a genome-wide high-resolution DamID binding map of histone H1 performed in Kc167 Drosophila cells (Braunschweig et al., 2009) identified the GAGAGA sequence as the binding sites for the transcription factors Trithorax-related (Trl/GAF), a shared motif for Non-stop-regulated genes also bound by H1. Moreover, GAGAGA sequence was also enriched in of Non-stop closed regions at the TSS of genes requiring Non-stop for expression (Figure 5F).

While Trl/GAF was not identified as part of the NIC in our proteomic purification, it associates in a protein complex containing Nup98, e(y)2 and Mod (mdg4) that regulate gene-expression (Pascual-Garcia et al. 2017). However, targetnig Trl/GAF or the adaptor protein CLAMP protein which bind to the GAGAGA sequence and associates with Cp190 and Mod (mdg4) did not result in loss of EC identity (Not shown; Bag et al. 2019). Therefore, we suggest that NIC is likely functionally and compositionally distinct from the Trl-containing complex.

Non-stop stabilizes NIC subunits, and Non-stop expression of in aged ECs restores largescale nuclear organization of ECs and suppresses aging phenotypes. As an isopeptidase, it seemed possible that Non-stop's ability to maintain expression of EC-related genes stems also from protecting NIC subunits from degradation. Indeed, the protein levels of Cp190, e(y)2, Mod (mdg4), and were reduced upon RNAi-dependent Non-stop elimination in young ECs as observed by immunostaining (Figure 6; Figure 6 Source data). Moreover, Nup98 was no-longer confined to the nuclear envelope but was localized to the nucleus interior in a punctate pattern (Figure 6E, F; Figure 6 Source data).

The observed changes in the stability of LamC and NIC subunits encouraged us to examine the larger organization of the nucleus using proteins that are markers for specific intranuclear domains and bodies. Loss of Non-stop in ECs resulted in decline in Coilin, which resides within Cajal bodies, and expansion in the expression of nucleolar Nop60B, a marker of the nucleolus. At the nuclear periphery we noted changes in localization of mTor and subsequent decline in 
LamC and reduced protein level of HP1b that is associated with heterochromatin and the chromocenter (Figure 6M-T; Figure 2G, H). Moreover, these changes were also observed upon targeting individual NIC subunits (Figure 6, Figure Supplemental 1).

As described above, the physiological relevance of a failure of Non-stop is significant to aging (Rodriguez-Fernandez et al. 2020). The cellular and tissue phenotypes associated with acute loss of Non-stop highly phenocopy aged midguts (Figure 2, Figure supplemental 2, 3). Indeed, a decline in the protein level of Non-stop was observed in aged ECs (Figure 7A, F, P). Moreover, the protein levels of NIC subunits CP190, e(y)2, and Mod (mdg4) were also reduced in aged ECs (compare Figure 7B-E to 7G-J; quantified in Figure 7P-T; Figure 7 source data). Thus, suggesting that a decline in Non-stop protein resulting in a failure to safeguard NIC stability accompanies aging. Therefore, we tested whether preventing the decline in Non-stop protein can protect the loss of NIC. Towards this aim we continuously expressed Non-stop in ECs using UAS-non-stop and the MyoIA $>$ Gal4/Gal80 ${ }^{\text {ts }}$ system, expressing Non-stop to a level similar to its expression in young ECs as determined by immunofluorescence (Fig 7). Indeed, and consistent with Non-stop's role as a key stabilizer of NIC expression of Non-stop, but not the control (UAS-LacZ), for five weeks prevented the aged dependent-decline of individual NIC subunits (Figure 7K-O; quantified in Figure 7P-T; Figure 7 source data).

We further examined whether maintaining Non-stop protein levels attenuates the aging of the midgut using the above system. Aging is associated with distorted nuclear organization of ECs (Figure 8, Figure 2, Figure 2 supplemental Figure 2). These changes include re-organization of the nuclear periphery, including a reduced level of LamC and histone $\mathrm{H} 1$ as well as redistribution of mTOR (Figure 8A-I). Aging is also associated with ectopic expression of LamDm0, and re-localization to the nuclear periphery of LamDm0 binding partner, Otefin (Ote) (Figure 8J, K, H, and Figure 8 supplemental Figure 1). Changes are also observed in the nucleus interior, involving the nucleolus and Cajal bodies as observed by the expansion of the nucleolar protein Nop60B and Coilin, which are resident proteins in these sub-nuclear bodies 
(Figure 8M-O; Figure 8 supplemental Figure 1). Consistent with Non-stop as a key identity supervisor relevant to aging, continuous expression of Non-stop for five weeks suppressed the above age-related changes in nuclear organization. Non-stop expression greatly restored LamC, histone $\mathrm{H} 1$ levels, localization of Mtor and suppressed the ectopic expression of LamDm0 and Ote, as well as restored the large-scale organization of the aged EC nucleus (Figure 8 C, F, I, L, $\mathrm{O}$ and Figure 8 supplemental Figure 1).

We further tested whether expression of Non-stop in wild-type ECs is capable of attenuating age-related changes in the gut epithelia. Indeed, expression of Non-stop suppressed classical characteristics of the aged midgut. For example, Non-stop expression maintained the expression of the EC marker Myo $>$ GFP and suppressed the ectopic expression of the ISC marker Delta PPCs (Figure 9A-D, H). Continuous expression of Non-stop in wildtype ECs for five weeks restored the expression of EC-related junctional proteins SSK and MESH, and suppressed the ectopic expression of Arm in PPCs (Figures 9E-G, and 9I-N). To test whether Non-stop maintenance affected the entire midgut at the organ level we tested for overall gut integrity using the Smurf assay. We observed that continuous expression of Non-stop in ECs greatly prevented the extensive leakage of blue-colored food observed in five weeks old aged animals restoring gut integrity (Figure 9O-Q). Thus, Non-stop is required for expression of ECgene programs, stabilizes NIC subunits in the adult, and together with NIC regulates large-scale organization of the differentiated nucleus, safeguarding EC identity and protecting from premature aging.

\section{Discussion:}

An in vivo screen identifies regulators that maintain the differentiated state. We performed an identity screen focused on conserved enzymes within the ubiquitin and ubiquitin-like pathways using the midgut tissue as a model system. Based on cell-specific secondary tests, 
we identified three categories of supervisors; 1 . EC-specific identity regulators 2 . Genes that are required for differentiated cell identity (both EEs and ECs) 3. Genes that are required for identity of all cell types (general identity regulators). Of specific interest were a group of genes (CG1490; CG2926; CG4080) whose elimination in EEs resulted in a loss of EC, but not EE, identity, acting as inductive identity regulators. Likely their effect on ECs involves cell cell communication via diffusible factors. The latter may be stochastic, or may be mediated by microtubule-based nanotubes as in the crosstalk between the hub cell (part of the stem cell niche) and stem cells in the Drosophila testis (Inaba et al. 2015).

The observation that Ub/UbL-related genes protect the differentiated identity is conserved across species. Screens in mammalian systems identified enzymes within the SUMO and ubiquitin pathways acting as a barrier against forced reprogramming of differentiated cell. Among these genes were Ubc9, the sole SUMO conjugating enzyme, that was also identified in our screen and the isopeptidase Psmd14 (Cheloufi et al. 2015; Buckley 2012). In addition to Non-stop, screen identified the iso-isopeptidase UTO6-like (CG7857), Usp7, and Rpn11 as regulators of EC identity. Rpn11 is part of the lid particle of the $26 \mathrm{~S}$ proteasome, involved in deubiquitinating proteins undergoing proteasomal degradation (Greene et al. 2020). We also identified the core particle proteasome subunit Pros- $\alpha 6$ (CG4904) as a bona-fide identity supervisor but not other proteasome subunits (that were also screened). Thus, it is not clear whether it represents a unique proteasome-independent function of the Pro- $\alpha 6$ subunit or whether Pro- $\alpha 6$ is a limiting subunit for proteasome biogenesis and activity in ECs.

Identity supervision is intimately involved in cancer, and genes regulating identity are likely to serve as a barrier to tumorigenesis and tumor suppressors. The human ortholog of Non-stop /USP22 has mixed oncogenic and tumor-suppressive functions (Jeusset et al. 2017). Relevant to our study is the observation that USP22 has tumor suppressive functions in colon cancer by reducing mTor activity (Kosinsky et al. 2020). Along this line it is interesting to note that 10/17 
of human orthologs to genes discovered in our screen are either mutated or silenced in cancer. Thus, future studies of these human orthologs may identify potent tumor suppressors in cancer.

Crosstalk between identity supervisors; Both Non-stop and the transcription factor Hey are bona-fide regulators of EC identity required for the expression of EC-related genes. We found a significant number of EC-related genes that required both Non-stop and Hey for their expression, suggesting that Hey and Not may co-regulate these genes. However, functional and epistatic tests suggest that Hey also acts upstream or in additional pathways to Non-stop. Hey binds to enhancers in lamin genes repressing the expression of the ISC-related lamin LamDm0 and enhances the expression of LamC. In contrast, Non-stop does not regulate the accessibility or expression of either LamDm0 or LamC at mRNA level. However, Non-stop is required for maintaining the levels of LamC protein. Therefore, loss of Non-stop result in a decline in LamC but not in the ectopic expression of LamDm0, which is observed upon acute loss of Hey or aging.

This discrepancy may be due to the presence of Hey on its repressed targets in young ECs where Non-stop is targeted, and directly repressing their expression as maybe in the case of LamDm0. Moreover, EC-specific expression of Non-stop did not suppress the phenotypes associated with acute loss of Hey in young ECs further supporting for Hey-dependent, but Non-stop independent functions. However, the ECs-specific expression of either Non-stop or Hey in aging midguts restores expression of LamC and repressed ectopic LamDm0 expression.

Potential function of Non-stop and the NIC: A possible function of the NIC may be the recruitment of the $\mathrm{H} 3 \mathrm{~K} 4 \mathrm{me} 2 / 3$ COMPASS methylases to catalyze $\mathrm{H} 3 \mathrm{~K} 9$ di- and trimethylation at enhancers and promotors, which are fundamental for gene activation (Shilatifard 2012; Sze and Shilatifard 2016). One prominent phenotype of loss of Non-stop was the mislocalization of Nup98 from the nuclear periphery to intranuclear punctate pattern. Nup98 was 
shown to recruit Set1 COMPASS to enhance histone H3K4me2-3 methylations in hematopoietic progenitors (Frank et al 2017). Thus, NIC may be required for recruitment of COMPASS and enhancing transcriptional memory promoting the transcription of EC-related genes.

$\mathrm{H} 3 \mathrm{~K} 4$ methylation and gene activation also require a ubiquitination and de-ubiquitination cycle catalyzed by the Bre1 ubiquitin ligase, and the de-ubiquitinase Non-stop/USP22 (Lee et al. 2007; Nakanishi et al. 2009). It is possible that with respect to EC-related genes, activity of Bre1 or Non-stop/NIC has a direct role in gene transcription in a similar fashion.

Moreover, we found that Non-stop is required for the stability of NIC, possibly by deubiquitinating NIC subunits in vivo, and that this stabilization is relevant in the context of physiological. However, the stabilization of NIC subunits maybe a more indirect role of Nonstop and mediated via LamC. We noticed that LamC expression partially restored the protein levels of NIC subunits and their intranuclear localization potentially by serving as a scaffold for NIC at the nuclear periphery. Thus, Non-stop may function at two levels; One is a direct role in transcription within NIC promoting de-ubiquitination of $\mathrm{H} 2 \mathrm{Bub}$ while a second function is the stabilization of identity supervisors including NIC subunits and LamC.

Not LLPs, and pre-mature aging: Changes in large-scale nuclear organization are hallmarks of aging (Zhang W 2020). Expression of identity supervisors can prevent age-related distortion of the nucleus EC identity and protect overall the epithelial tissue (This work and flint Brodsly et al 2019). However, to accomplish this, Non-stop or Hey were continuously expressed in ECs and temporal expression of Hey or Non-stop in already aged ECs was not sufficient to suppress aging phenotypes. Thus, if the levels of identity supervisors are kept at youthful levels, they can continue to maintain cell identity and prevent signs of aging, effectively keeping the gut organization and structure similar to young tissue (Kenyon 2010).

Furthermore, it is not clear how expression of a single regulator like Non-stop has an extensive impact on the entire nucleus. Recent studies suggest that Non-stop functions in additional 
multiprotein complexes that may regulate large-scale cellular organization. For example, Nonstop is part of an Arp2/3 and WAVE regulatory (WRC) actin-cytoskeleton organization complex where it deubiquitinates the subunit SCAR (Cloud et al. 2019). In this regard, a nuclear actin organizing complex, WASH, interacted with nuclear Lamin and was required for large scale nuclear organization (Varbooon et al. 2015). Thus, it is tempting to suggest that such complexes are required to maintain cell identity, and that subunits within these complexes are deubiquitinated by Non-stop.

Recent studies suggest that the organization of the nucleus is mediated by the biophysical properties of the nuclear protein milieu and interaction with macro-molecules such as chromatin and formation of local condensates (Strom and Brangwynne 2019; Yoshizawa 2020). These biophysical forces including liquid-liquid phase-separation (LLPS), are critical for compartmentalization of the nucleus, heterochromatin and euchromatin formation, establishment of transcription factories and intranuclear bodies. Thus, Non-stop/NIC activity may be critical for safeguarding the stability of proteins that their local concentration is critical for the self-organization and compartmentalization of the differentiated nucleus.

In this regard many nuclear proteins are extremely long-lived proteins (LLPs) among them are nuclear pore complex proteins (NPCs) and core histones (Toyama et al. 2013; Toyama 2019). The extended stability of LLPs may originate from intrinsic properties of LLPs, or due to sequestration and evading degradation. However, increased stability may be also actively maintained by constitutive de-ubiquitination. Indeed, post-transcriptional modification by ubiquitin and SUMO were shown to regulate lamin stability and their intranuclear localization (Blank M. 2020). Specifically, type-A lamin and its splice variant Progerin, the cause of Hutchinson Gilford progeria syndrome (HGPS), a premature aging syndrome, are degraded by the HECT-type E3 ligase Smurf2 via ubiquitin-dependent autophagy (Borroni et al. 2018). The elimination of Progerin by expression of Smurf2 in HGSP-fibroblasts reduced the deformation observed in these cells. Thus, it is possible that enhancing Progerin degradation by inhibiting 
the human ortholog of Non-stop, USP22, will restore nuclear architecture, and suppress the premature aging phenotypes observed in HGPS cells.

Authors' contributions: NE, LI, ELB, OM RM PV, TD and AO designed and preformed experiments, WW and NE preformed genomic and bioinformatics analyses. All authors analyzed data. ELB, RM, TD, and AO wrote the paper.

Acknowledgments: We are grateful for mass spectrometry done by Skylar Martin-Brown, Laurence Florens, and Michael P Washburn at the Stowers Institute. We would like to thank Sarah Bray, Adi Salzberg, Bruce Edgar, Jeff Reinitz, Lori Wallrath, Pamela Geyer, Yossi Greenbaum, Lorry Pile, Erika Matunis, Díaz-Benjumea, Mikio Furuse Bas Van-Steensel, Moshe Oren, Joseph Gall, the Bloomington, VDRC, and NIG-FLY Drosophila stock centers for sharing antibodies, fly lines, reagents, and data.

This research was supported by: The School of Biological and Chemical Sciences, UMKC; University of Missouri Research Board; UMKC SEARCH, UMKC SUROP scholars programs, and NIH Academic Development Via Applied and Cutting Edge Research (ADVANCER) program; NIGMS grant 5R35GM118068 to RM. Washington University School of Medicine / St. Louis Children's Hospital Children's Discovery Institute, MC-II-2014-363 to TD. Russian Science Foundation 19-74-30026 to PG, and the Israel Science Foundation (ISF) (Grants 739/15, 318/20), and by the Flinkman Marandi Family cancer research grant to AO. 


\section{References:}

Abed, M., Barry, K. C., Kenyagin, D., et al. (2011) Degringolade, a SUMO-targeted ubiquitin ligase, inhibits Hairy/Groucho-mediated repression. EMBO J. 20:1289-1301. doi:10.1038/emboj.2011.42

Bag I, Dale RK, Palmer C, Lei EP. (2019) The zinc-finger protein CLAMP promotes gypsy chromatin insulator function in Drosophila. J Cell Sci.;132(5):jcs226092.

doi:10.1242/jcs.226092

Bensellam M., Jonas J. C., Laybutt, D.R., (2018) Mechanisms of $\beta$-cell dedifferentiation in diabetes: recent findings and future research directions. J Endocrinol. 236:R109-R143. doi:10.1530/JOE-17-0516

Biteau, B., Karpac, J., Supoyo, S., Degennaro, M., Lehmann, R., and Jasper, H. (2010). Lifespan extension by preserving proliferative homeostasis in Drosophila. PLoS Genet. 6:e1001159. doi:10.1371/journal.pgen.1001159

Bitman-Lotan, E., Orian, A., (2018) Chromatin, Nuclear Lamins, and maintenance of the differentiated identity. Current Opinion in System Biology 11, 1-8.

Blank M. (2020) Targeted Regulation of Nuclear Lamins by Ubiquitin and Ubiquitin-Like Modifiers. Cells.;9(6):1340. doi:10.3390/cells9061340

Blau, H.M., and Baltimore, D. (1991). Differentiation requires continuous regulation. J Cell Biol. 112, 781-783. doi:10.1083/jcb.112.5.781

Bonnay, F., Cohen-Berros, E., Hoffmann, M., Kim, S. Y., Boulianne, G. L., Hoffmann, J.A., Matt, N., Reichhart, J. M. (2013) big bang gene modulates gut immune tolerance in Drosophila. Proc Natl Acad Sci U S A. 110:2957-2962 doi:10.1073/pnas.1221910110

Booth LN, Brunet A. (2016) The Aging Epigenome. Mol Cell. 62:728-744 doi:10.1016/j.molcel.2016.05.013 
Borroni AP, Emanuelli A, Shah PA, et al. (2018) Smurf2 regulates stability and the autophagic-lysosomal turnover of lamin A and its disease-associated form progerin. Aging Cell.;17(2):e12732. doi:10.1111/acel.12732

Brand, A.H., and Perrimon N. (1993). Targeted gene expression as a means of altering cell fates and generating dominant phenotypes. Development 118, 401-415.

Braunschweig U, Hogan GJ, Pagie L, van Steensel B. (2009) Histone H1 binding is inhibited by histone variant H3.3. EMBO J. 28:3635-3645. doi:10.1038/emboj.2009.301

Buchon, N., Osman, D., David, F.P., Fang, H.Y., Boquete, J.P., Deplancke, B., and Lemaitre, B. (2013). Morphological and molecular characterization of adult midgut compartmentalization in Drosophila. Cell Rep. 3, 1725-1738. doi:10.1016/j.celrep.2013.04.001

Buckley SM, Aranda-Orgilles B, Strikoudis A, et al. (2012) Regulation of pluripotency and cellular reprogramming by the ubiquitin-proteasome system. Cell Stem Cell 11:783-798. doi:10.1016/j.stem.2012.09.011

Buenrostro JD, Giresi PG, Zaba LC, Chang HY, Greenleaf WJ. (2013) Transposition of native chromatin for fast and sensitive epigenomic profiling of open chromatin, DNA-binding proteins and nucleosome position. Nat Methods.;10:1213-1218. doi:10.1038/nmeth.2688

Cappadocia, L., Lima, C. D. (2018) Ubiquitin-like Protein Conjugation: Structures, Chemistry, and Mechanism. Chem Rev. 118:889-918 doi:10.1021/acs.chemrev.6b00737 Cheloufi S, Elling U, Hopfgartner B, et al (2015) The histone chaperone CAF-1 safeguards somatic cell identity. Nature 528:218-224. doi:10.1038/nature15749

Chen, H., Zheng, X., Zheng, Y.(2014) Age-associated loss of lamin-B leads to systemic inflammation and gut hyperplasia. Cell 159:829-843. doi:10.1016/j.cell.2014.10.028

Chua, B.A., Signer, R. A. J. (2020) Hematopoietic stem cell regulation by the proteostasis network. Curr Opin Hematol. 27:254-263. doi:10.1097/MOH.0000000000000591

Cloud V, Thapa A, Morales-Sosa P, et al. (2019) Ataxin-7 and Non-stop coordinate SCAR protein levels, subcellular localization, and actin cytoskeleton organization. Elife.8:e49677. doi:10.7554/eLife.49677

Conway, E., Healy ,E., Bracken, A. P. (2015) PRC2 mediated H3K27 methylations in cellular identity and cancer. Curr Opin Cell Biol 37:42-48. doi:10.1016/j.ceb.2015.10.003 
Deneris, E.S., and Hobert, O. (2014). Maintenance of postmitotic neuronal cell identity. Nat Neurosci. 17, 899-907. doi:10.1038/nn.3731

Enam, C., Geffen, Y., Ravid, T., Gardner RG. (2018) Protein Quality Control Degradation in the Nucleus. Annu Rev Biochem. 2018;87:725-749. doi:10.1146/annurev-biochem-062917012730

Evans, C. J., Olson J. M., Ngo, E. Kim, K. T, Lee, N. E., Kuoy, E. Patananan, A. N., Sitz, D., Tran, P., Do, M T., Yackle, K., Cespedes, A., Hartenstein, V., Call, G. B., Banerjee, U. (2009) G-TRACE: rapid Gal4-based cell lineage analysis in Drosophila Nature Methods 6:603-605. doi:10.1038/nmeth.1356

Ferrandon D. (2013) The complementary facets of epithelial host defenses in the genetic model organism Drosophila melanogaster: from resistance to resilience. Curr Opin Immunol. 25:59-70. doi:10.1016/j.coi.2012.11.008

Flint Brodsly N, Bitman-Lotan E, Boico O, et al (2019) The transcription factor Hey and nuclear lamins specify and maintain cell identity. Elife. https://doi.org/10.7554/elife.44745 doi:10.7554/eLife.44745

Franks TM, McCloskey A, Shokirev MN, Benner C, Rathore A, Hetzer MW. (2017) Nup98 recruits the Wdr82-Set1A/COMPASS complex to promoters to regulate H3K4 trimethylation in hematopoietic progenitor cells. Genes Dev.31:2222-2234. doi:10.1101/gad.306753.117

Fyodorov DV, Zhou BR, Skoultchi AI, Bai Y. Emerging roles of linker histones in regulating chromatin structure and function. Nat Rev Mol Cell Biol. 2018;19(3):192-206.

doi:10.1038/nrm.2017.94

Golovnin, A., Mazur, A., Kopantseva, M., Kurshakova, M., Gulak, P. V., Gilmore, B., Whitfield, W. G., Geyer, P., Pirrotta, V. and Georgiev, P. (2007). Integrity of the Mod (mdg4)67.2 BTB domain is critical to insulator function in Drosophila melanogaster. Mol. Cell. Biol. 27, 963-974 doi:10.1128/MCB.00795-06

Gruenbaum Y, Foisner R. (2015) Lamins: nuclear intermediate filament proteins with fundamental functions in nuclear mechanics and genome regulation. Annu Rev Biochem.;84:131-164. doi:10.1146/annurev-biochem-060614-034115

Greene ER, Dong KC, Martin A. (2020) Understanding the 26S proteasome molecular machine from a structural and conformational dynamics perspective. Curr Opin Struct Biol. 61:33-41. doi:10.1128/MCB.00795-06

Heideker, J., Wertz, I. E. (2015) DUBs, the regulation of cell identity and disease.

Biochem J. 465:1-26 doi:10.1042/BJ20140496 
Hnisz, D., Abraham, B. J,. Lee, T. I., Lau, A., Saint-André, V., Sigova, A. A,. Hoke, H. A., Young, R. A. (2013) Super-enhancers in the control of cell identity and disease. Cell. 155:934-947. doi:10.1016/j.cell.2013.09.053

Holmberg, J., and Perlmann, T. (2012). Maintaining differentiated cellular identity. Nat Rev Genet. 13, 429-439. doi:10.1038/nrg3209

Höhfeld J, Hoppe T. (2018) Ub and Down: Ubiquitin Exercise for the Elderly. Trends Cell Biol.;28:512-522. doi:10.1016/j.tcb.2018.03.002

Hudish, L. I., Resch, J. E., Sussel, L. J. (2019) $\beta$ Cell dysfunction during progression of metabolic syndrome to type 2 diabetes. Clin Invest. 129:4001-4008 doi:10.1172/JCI129188

Hung, R. J., Hu, Y., Kirchner, R., Liu, Y., Xu, C., Comjean, A., Tattikota, S. G., Li, F., Song, W., Ho, Sui. S., Perrimon, N. (2020) A cell atlas of the adult Drosophila midgut .Proc Natl Acad Sci U S A. 117:1514-1523 doi:10.1073/pnas.1916820117

Inaba M, Buszczak M, Yamashita YM. (2015) Nanotubes mediate niche-stem-cell signalling in the Drosophila testis. Nature 523:329-332. doi:10.1038/nature14602

Jasper H. (2020) Intestinal Stem Cell Aging: Origins and Interventions.Annu Rev Physiol. 82:203-226. doi:10.1146/annurev-physiol-021119-034359

Jeusset LM, McManus KJ (2017); Ubiquitin Specific Peptidase 22 Regulates Histone H2B Mono-Ubiquitination and Exhibits Both Oncogenic and Tumor Suppressor Roles in Cancer. Cancers. 9(12):167. doi:10.3390/cancers9120167

Jiang, H., and Edgar, B.A. (2012). Intestinal stem cell function in Drosophila and mice. Curr Opin Genet Dev. 22, 354-360. doi:10.1016/j.gde.2012.04.002

Kevei, É., Hoppe, T. (2014). Ubiquitin sets the timer: impacts on aging and longevity. Nat Struct Mol Biol 21, 290-292 https://doi.org/10.1038/nsmb.2806

Kenyon, C. J (2010). The genetics of ageing Nature.464: 504-512. doi:10.1038/nature08980

Korzelius, J., Naumann, S.K., Loza-Coll, M.A., Chan, J.S., Dutta, D., Oberheim, J., Gläßer, C., Southall, T.D., Brand, A.H., Jones, D.L, and Edgar, B.A. (2014). Escargot maintains stemness and suppresses differentiation in Drosophila intestinal stem cells. EMBO J. 33, 2967-2982. doi:10.15252/embj.201489072 
Kosinsky RL, Zerche M, Saul D, et al. (2020) USP22 exerts tumor-suppressive functions in colorectal cancer by decreasing mTOR activity. Cell Death Differ.;27(4):1328-1340. doi:10.1038/s41418-019-0420-8

Lemaitre, B., and Miguel-Aliaga, I. (2013). The digestive tract of Drosophila melanogaster. Annu Rev Genet. 47, 377-404. doi:10.1146/annurev-genet-111212-133343

Lee JS, Shukla A, Schneider J, et al. (2007) Histone crosstalk between H2B monoubiquitination and H3 methylation mediated by COMPASS. Cell. 131:1084-1096. doi:10.1016/j.cell.2007.09.046

Li X, Seidel CW, Szerszen LT, Lange JJ, Workman JL, Abmayr SM. (2017) Enzymatic modules of the SAGA chromatin-modifying complex play distinct roles in Drosophila gene expression and development. Genes Dev.;31:1588-1600. doi:10.1101/gad.300988.117

Martin KA, Poeck B, Roth H, Ebens AJ, Ballard LC, Zipursky SL. (1995) Mutations disrupting neuronal connectivity in the Drosophila visual system. Neuron.;14:229-240. doi:10.1016/0896-6273(95)90281-3

Mohan, R. D., Dialynas, G., Weake V. M., Liu, .J, Martin-Brown, S., Florens, L., Washburn, M. P., Workman, J. L., Abmayr, S. M. (2014) .Loss of Drosophila Ataxin-7, a SAGA subunit, reduces H2B ubiquitination and leads to neural and retinal degeneration. Genes Dev28:259-272 doi:10.1101/gad.225151.113

Monastirioti M, Giagtzoglou N, Koumbanakis KA, et al. (2010) Drosophila Hey is a target of Notch in asymmetric divisions during embryonic and larval neurogenesis. Development.;137:191-201. doi:10.1242/dev.043604

Morgan, M. T., Haj-Yahya, M., Ringel, A. E., Bandi, P., Brik, A., Wolberger, C. (2016) Structural basis for histone H2B deubiquitination by the SAGA DUB module. Science. 351:725-728 doi:10.1126/science.aac5681

Morgan MT, Wolberger C. (2017) Recognition of ubiquitinated nucleosomes. Curr Opin Struct Biol. ;42:75-82. doi:10.1016/j.sbi.2016.11.016

Nakanishi S, Lee JS, Gardner KE, et al. (2009) Histone H2BK123 monoubiquitination is the critical determinant for H3K4 and H3K79 trimethylation by COMPASS and Dot1. J Cell Biol.;186(3):371-377. doi:10.1083/jcb.200906005

Natoli, G. (2010). Maintaining cell identity through global control of genomic organization. Immunity 33, 12-24. doi:10.1016/j.immuni.2010.07.006 
Naetar, N., Ferraioli, S., Foisner, R. (2017) Lamins in the nuclear interior - life outside the lamina. J Cell Sci. 130:2087-2096 doi:10.1242/jcs.203430

Neves, J., Demaria, M., Campisi, J., and Jasper, H. (2015). Of flies, mice, and men: Evolutionarily conserved tissue damage responses and aging. Dev Cell 32, 9-18. Neves, J., Demaria, M., Campisi, J., and Jasper, H. (2015). doi:10.1016/j.devcel.2014.11.028 doi:10.1016/j.molcel.2017.02.020

Rera, M., Clark, R.I., and Walker, D.W. (2012). Intestinal barrier dysfunction links metabolic and inflammatory markers of aging to death in Drosophila. Proc Natl Acad Sci. USA 109, 21528-21533. doi:10.1073/pnas.1215849110. doi:10.1073/pnas.1215849110

Rodriguez-Fernandez I. A., Tauc H. M. , Jasper , H. (2020) Hallmarks of aging Drosophila intestinal stem cells Mech Ageing Dev. 2020;190:111285 doi:10.1016/j.mad.2020.111285

Salmeron, J.M., Leuther, K.K., and Johnston, S.A. (1990). GAL4 mutations that separate the transcriptional activation and GAL80-interactive functions of the yeast GAL4 protein. Genetics $125,21-27$.

Shaw, R.L., Kohlmaier, A., Polesello, C., Veelken, C., Edgar, B.A., Tapon, N. (2010) The Hippo pathway regulates intestinal stem cell proliferation during Drosophila adult midgut regeneration. Development.137:4147-4158. doi:10.1242/dev.052506

Sze CC, Shilatifard A. (2016) MLL3/MLL4/COMPASS Family on Epigenetic Regulation of Enhancer Function and Cancer. Cold Spring Harb Perspect Med. 6:a026427 doi:10.1101/cshperspect.a026427

Shilatifard A (2012). The COMPASS family of histone H3K4 methylases: mechanisms of regulation in development and disease pathogenesis. Annu Rev Biochem.;81:65-95. doi:10.1146/annurev-biochem-051710-134100

Strom AR, Brangwynne CP. (2019) The liquid nucleome - phase transitions in the nucleus at a glance. J Cell Sci. 132:jcs235093. doi:10.1242/jcs.235093

Song, L., Luo, Z. Q. (2019) Post-translational regulation of ubiquitin signaling. J Cell Biol. 218:1776-1786 doi:10.1083/jcb.201902074

Swatek, K. N., Komander, D. (2016) Ubiquitin modifications. Cell Res. 26:399-422. doi:10.1038/cr.2016.39 
Takeda, K., Okumura, T., Taniguchi, K., Adachi-Yamada, T.. (2018) Adult Intestine Aging Model. Adv Exp Med Biol. 1076: 11-23 doi:10.1007/978-981-13-0529-0_2

Tata, P.R., and Rajagopal, J. (2016). Cellular plasticity: 1712 to the present day. Curr Opin Cell Biol. 43, 46-54. doi:10.1016/j.ceb.2016.07.005

Toyama BH, Savas JN, Park SK, et al. (2013) Identification of long-lived proteins reveals exceptional stability of essential cellular structures. Cell.;154(5):971-982 doi:10.1016/j.cell.2013.07.037

Toyama BH, Arrojo E Drigo R, Lev-Ram V, et al. (2019) Visualization of long-lived proteins reveals age mosaicism within nuclei of postmitotic cells. J Cell Biol. 218:433-444. doi:10.1083/jcb.201809123

Verboon JM, Rincon-Arano H, Werwie TR, et al. Wash interacts with lamin and affects global nuclear organization. Curr Biol. 2015;25(6):804-810. doi:10.1016/j.cub.2015.01.052

Vilchez D, Saez I, Dillin A (2014). The role of protein clearance mechanisms in organismal ageing and age-related diseases. Nat Commun.;5: 5659. doi:10.1038/ncomms6659

Washburn MP, Wolters D, Yates JR 3rd. (2001) Large-scale analysis of the yeast proteome by multidimensional protein identification technology. Nat Biotechnol.;19(3):242-247. doi:10.1038/85686

Weake VM, Lee KK, Guelman S, Lin CH, Seidel C, Abmayr SM, Workman JL (2008) SAGA-mediated H2B deubiquitination controls the development of neuronal connectivity in the Drosophila visual system. EMBO J. 27:394-405 doi:10.1038/sj.emboj.7601966

Yau, T.Y., Molina, O., Courey, A.J. (2020) SUMOylation in development and neurodegeneration. Development. 147:dev175703. doi:10.1242/dev.175703

Yoshizawa T, Nozawa RS, Jia TZ, Saio T, Mori E. (2020) Biological phase separation: cell biology meets biophysics. Biophys Rev. 12:519-539. doi:10.1007/s12551-020-00680-x

Zhang, X. Y., Varthi, M., Sykes, S. M., Phillips, C., Warzecha, C., Zhu, W., Wyce, A., Thorne, A.W., Berger, S. L., McMahon, S. B (2008) The putative cancer stem cell marker USP22 is a subunit of the human SAGAcomplex required for activated transcription and cellcycle progression. Mol Cell. 2008 Jan 18;29(1):102-11. doi:10.1016/j.molcel.2007.12.015

Zhang W, Qu J, Liu GH, Belmonte JCI. (2020) The ageing epigenome and its rejuvenation. Nat Rev Mol Cell Biol. 21:137-150. doi:10.1038/s41580-019-0204-5 


\section{Figure legends}

\section{Figure 1: RNAi Screen identified Non-stop (Not) as an ECs identity supervisor. (A)}

Schematic diagram of midgut differentiation and an outline of the $\mathrm{Ub} / \mathrm{UbL}$ screen (see text for details). The Notch ligand, Delta, is expressed on the surface of Intestinal stem cells (ISC) marked in red. (B) Phenotypes expected from positive hits: 1. Loss of expression of EC-specific GFP (expressed only in fully differentiated ECs using MyoIA $>$ Gal4/Gal80 $0^{\text {ts }}$ system), along with ectopic expression of the ISC marker Delta (red). 2. Polyploid cells that ectopically express Delta and retain expression of GFP. (C-F) Confocal images using UAS-LacZ (C, E) or UASNot RNAi (D, F) along with UAS-GFP expressed under the control of MyoIA $>\mathrm{Ga} 14 / \mathrm{Gal} 80^{\text {ts }}$ system. Scale bar is $10 \mu \mathrm{M}$. The stem cell marker Delta (C, D) and EE marker Prospero (E, F) are shown in red. $(\mathbf{G}, \mathbf{H})$ Quantification of three biological repeats of experiments similar to that shown in C-F. *(I, J) Expression of UAS-Not RNAi, but not control, in ECs for 48 h using MyoIA $>$ Gal4/Gal80 ${ }^{\text {ts }}$ results in ectopic expression of the Notch-reporter (red) in polyploid cells. (K, L, Q) Expression of the escargot progenitor enhancer reporter M5-4-LacZ in control or Not-targeted ECs (red). Yellow arrows points to cells shown in the insets. White arrows in L are examples of EC-like polyploid cells ectopically expressing the reporter. (M, N) Loss of Not in ECs resulted in an increase in the mitosis marker p-H3 in small cells. (O, P) Loss of Not in ECs impairs gut integrity as evident by the leakage of blue-colored food into the abdomen (smurf assay); $24 \%$ of Not-RNAi flies show loss of gut integrity versus $0 \%$ in control 
flies ( $\mathrm{n}=44,55$ respectively, $\mathrm{p}<0.001)(\mathbf{Q})$ Quantification of M5-4 positive PPCs in control and upon targetnig Not in ECs. $(* * *=p<0.001 * * p<0.01)$. (R) Survival analysis of flies expressing the indicated transgenes in ECs under the control of MyoIA-Gal4/Gal80ts $(* * * *=\mathrm{p}<0.0001)$.

Figure 1; Figure Supplemental 1: Examples of positive hits of the Ub/UbL screen: (A-K) Confocal images of the midgut tissue and the indicated transgenes expressed in ECs using the MyoIA-Gal4/Gal80ts. White arrows indicate cells shown in insets. Scale bar is $10 \mu \mathrm{M}$ MyoIA>UAS-GPF marks fully differentiated ECs, Delta is shown in red, and DAPI (blue) marks DNA. (A-G) Examples of positive hits from the screen. (H-J) Loss of in ECs Non-stop using three independent UAS-RNAi transgenic lines results in loss of EC identity.

Figure 1 Figure Supplemental 2: Characterization of Non-stop in midgut cells (A-G) Confocal images of the midgut tissue and the indicated transgenes expressed in EC using the MyoIA-Ga14/Gal80ts. (A-D) Not is expressed in all mid gut cells. Expression of endogenous Not protein (red) was tested relative to the expression of UAS-GFP that was expressed under the cell-specific GAL4 drivers: Dl>GAL4 (ISC); $\mathrm{Su}(\mathrm{H})>$ Gal4 (EBs); Prospero Gal4 (EE's) and MyoIA $>$ Gal4 (ECs). Arrow point to cells shown in insets. (E) Expression of Not upon activating UAS-Not-RNAi in ECs. (F, G) Level of H2Bub (red) in midguts expressing the control (F), or UAS-Not-RNAi (G) in ECs using the MyoIA>Gal4, UAS-GFP system. Western-blot analysis of $\mathrm{H} 2 \mathrm{Bub}$ and $\mathrm{H} 2 \mathrm{~B}$ in midgut derived extracts of the indicated genotypes. Actin serves as a loading control. (I-L) Expression of Delta (I, J, red) or Prospero (K, L, red) in midguts expressing control $(\mathrm{I}, \mathrm{K})$ or the UAS-Not RNAi $(\mathrm{J}, \mathrm{L})$ in EE using prospero $>$ GAL4 $4^{\text {ts }}$, UAS-GFP system. Scale bar is $10 \mu \mathrm{M}$

Figure 1; Supplemental Table 1: Summary of the screen Ub/UbL screen results. 
Figure 1; Source data: Quantification of data presented in Figure 1G, H.

Figure 2: G-TRACE-Lineage characterization of Not targeted young ECs and, aged ECs. (A) Schematic diagram of EC-G-TRACE-lineage tracing system adopted from (Flint-Brodsly 2019). PPC** (RFP-GFP ${ }^{+}$), are EC that are no-longer differentiated. PPC* (RFP-GFP-) are miss-differentiated progenitors. (B-O) Confocal microscopy of midguts expressing the indicated transgenes, under the control of MyoIA ${ }^{\text {ts }}$ G-TRACE system using the indicated antibodies. DAPI (blue) marks DNA. Arrows point to cells shown in the insets with individual far-RFP, RFP and GFP channels. DAPI (blue) marks DNA scale bar is $10 \mu \mathrm{M}$.

(B-G) G-TRACE of EC in control young midgut expressing either UAS-LacZ (B, D, F), or UAS-Not- RNAi (C, E, G). Arrows point to cells shown in the insets with individual far-RFP, RFP and GFP channels. (H) G-TRACE-based quantification of PPC types (wildtype, PPC*

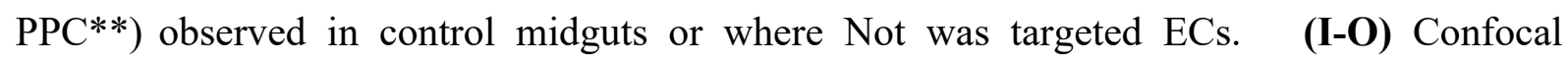
microscopy of midguts expressing MyoIA ${ }^{\text {ts }}>$ G-TRACE system using the indicated antibodies. (B, J, L, N) G-TRACE of EC in young, and (I, K, M, O) old midguts. (P). Quantification of indicated PPCs expressing Odd-Skipped, and Delta similar to experiments shown in L-O.

Figure 2; Figure Supplemental 1: Non-stop is required for expression of EC transcription factors Caudal and Pdm1. (A-D) Confocal images of the midgut tissue and the indicated transgenes expressed in EC using the MyoIA-Gal4/Gal80ts. (A, B) anti-Pdm1 (C, D) antiCaudal Arrows point to cells shown in the inset. Scale bar is $10 \mu \mathrm{M}$

Figure 2; Figure Supplemental 2: Miss-regulation of enhancers activity and nuclear Lamins in aged enterocytes. (A-N) Confocal images with the indicated antibodies of adult Drosophila midgut epithelium expressing the indicated transgenes, DAPI marks DNA, and scale bar is $10 \mu \mathrm{m}$. (A-H) transgenic lines expressing UAS-GFP under the control of the EC- 
specific promoter MyoIA-GAL4/Gal80 ${ }^{\text {ts }}$ system. (I-P) Transgenic lines expressing G-TRACE system under the control of the enterocyte specific promoter MyoIA ${ }^{\text {ts }}$ system. The expression of M5-4::LacZ stem cells enhancer of esg gene is shown in A, B, I, and J. The protein level and distribution of the indicated protein is shown; LamC expression (C, D, K, L). Lamin Dm0 (LamDm0), E, F, M, N., and Otefin (Ote) (G, H, O, P). Otefin (Ote) protein level in control young ECs or old $(\mathrm{G}, \mathrm{H})$.

Figure 2; Figure Supplemental 3: Hallmarks of aging in the Drosophila midgut (A-L Confocal images of indirect immunofluorescence staining with the indicated antibodies of adult Drosophila midgut intestinal epithelium expressing termed MyoIA ${ }^{\text {ts }}$. Scale bar is $10 \mu \mathrm{m} .(\mathrm{A}, \mathrm{C}$, E, G, I, K): Young mid-guts (four days old adults). (B, D, F, H, J, L) Four weeks old guts. DAPI marks DNA and arrows indicates cells shown in the insets. SSK; Snake Skin; Arm, Armadillo; Dlg, Disc large.

Figure 2 Source data file: Quantification data for Figures 2H, 2P,

Figure 3: Identification of a Non-stop-identity complex (NIC). (A-C) Purification scheme of nuclear Not-associated complexes from Drosophila S2 cells. (see text and methods; adopted from Cloud et al 2019) (B) Identification of Not-associated isopeptidase activity proteins by immunoprecipitation followed by size fractionation and mass-spectrometry. CP190, Mod (mdg4), Nup96-98, and E(y)2 were all present in Group 2. Not-FH; IP with full length Not FLAG-HA tagged (C) Summary of protein complexes isolated identified by mass-spectrometry (D, E) Not binds to the C-terminal portion of CP190 and to E(y)2. (D) Western-blot of in vitro binding between HA-Not derived from S2 cell extract and the indicated bacterially expressed purified His-tagged proteins. 10\% input is shown. (E) Comassie blue staining of the indicated bacterially expressed His-tagged proteins used in the binding assay in (D). (F) Schematic 
diagram of Y2H interaction assay between CP190 and Non-stop. Different fragments of CP190 were fused to the activation domain (AD) of GAL4 and tested for interaction with Non-stop fused to the DNA-binding domain (BD) of GAL4. Protein domains of full-length CP190 are indicated as boxes, and lines represent the different deletion fragments. Zf denote zinc-fingers; BTB, BTB/POZ domain; D, aspartic acid -rich region; M, microtubule-interacting region; E, acid glutamate-rich region of CP190. The results are summarized in columns on the right (BDNot and BD alone), with the "+” and "-“" signs denotes presence and absence of interaction, respectively.

Figure 3; Figure Supplemental 1: Loss of SAGA subunits and $\mathrm{Su}(\mathrm{Hw})$ does-not affect EC identity. (A-E) Confocal images of the midgut tissue and the indicated transgenes expressed in EC using the MyoIA-Ga14/Gal80ts. (A-E) anti-Delta (A'-C') anti-Odd-skipped. (A, A') UASLacZ, (B, B') UAS-Sgf11-RNAi; (C, C') UAS GCN5-RNAi, (D) UAS-Atx7 RNAi (E) UAS$\mathrm{Su}(\mathrm{Hw})$ - RNAi. DAPI marks DNA, Scale bar is $10 \mu \mathrm{M}$.

Figure 4: Not identity complex (NIC) regulates EC identity. Confocal images of the midgut tissue using the indicated antibodies; (A-E) LamC, (G-K) Delta, DAPI marks DNA (blue). The indicated transgenes were expressed in EC using the MyoIA-Gal4/Gal80ts system for fortyeight hours. UAS-LacZ (A, G) UAS-e(y)2-RNAi (B, H); UAS-CP190-RNAi; (C, I). Nup98$96(\mathrm{D}, \mathrm{J})$ Mod (mdg4) (E, K) White arrows points to cells shown in insets, and scale bar is $10 \mu \mathrm{M}$. Quantification is shown in (F) for LamC. and (L) for Delta.

Figure 4 Supplemental source data : Quantification of cell populations described in 4F, 4L.

Figure 5: Not regulates EC-gene expression and is required for chromatin accessibility. (A) Venn diagram comparing EC-related genes (Blue; Korzelius 2014), genes exhibiting 
reduced expression upon loss of Not in ECs (Green), and chromatin regions with reduced accessibility upon loss of Not in ECs identified by ATAC-seq (Orange). (B) Metascape analysis of Not-down-regulated genes in ECs. (C) Venn diagram comparison of genes that exhibit reduced expression upon loss of either Not or Hey in ECs, as well as genes in the vicinity of regions showing reduced accessibility upon loss of Not. (D) Venn diagram of genes that exhibit reduced expression upon loss of Not or Hey and of genes with reduced expression upon over expression of LamDm0 in ECs (E) GO analysis of genes downregulated by loss of Not in ECs exhibiting reduced accessibility. Observed gene count; number of genes identified from this group in both ATAC-seq and RNA-seq (F) Genome-wide alignment and MEME analysis of regions with reduced accessibility in the vicinity of down-regulated genes upon loss of Not in ECs. TSS, transcriptional start site; TES, transcription end site. (G-H) Confocal images of the midgut tissue using $\alpha$-Histone $\mathrm{H} 1$ (red), and expressing the indicated transgenes in ECs using the MyoIA-Gal4/Gal80ts system for forty-eight hours, DAPI marks DNA (blue). (G) UASLacZ (control) (H,) UAS-Not RNAi. Scale bar is $10 \mu \mathrm{M}$. (I, J) western-blot analysis of the indicated proteins derived from gut extract (I), or S2 Drosophila cell extract (J) Histone H3 and Actin serve as loading controls.

\section{Figure 5; Figure Supplemental 1: Analysis of Not-related RNA-seq and ATAC-seq.}

(A) Venn diagram comparing genes exhibiting enriched expression in differentiated gut cells (Blue), genes exhibiting reduced expression upon loss of Not in ECs (Green), and chromatin regions with reduced accessibility upon loss of Not in ECs identified by ATAC-seq (Orange). Venn diagram comparison of genes that exhibit reduced expression upon loss of either Not or expression of LamDm0 ECs, and EC-expressed genes (B) Venn diagram comparison of genes that exhibit reduced expression or accessibility upon loss of Not, and genes with reduced expression upon over expression of LamDm0. (C) Venn diagram comparison of genes that 
exhibit upregulation in expression upon loss of either Not or Hey in ECs or over-expression of LamDm0 in ECs. (D). Three principle components analysis of RNA-seq.

(E-G) Expression of LamC suppresses the ectopic expression of PNCA in EC that are no longer differentiated (PCC**). Confocal images of the midgut tissue using the indicated antibodies. (E-F) G-TRACE analysis; (E) Control ECs (expressing UAS-LacZ) do not express PCNA, and are both $\mathrm{RFP}^{(+)} \mathrm{GFP}^{(+)}$. (F) $\mathrm{PPC}^{* *}$ are $\mathrm{GFP}^{(+)}$and $\mathrm{RPF}^{(-)}\left(\mathrm{PPC}^{* *}\right)$ and express PCNA (purple). Arrow points to cells shown in the insets (individual channels). (G) ECs where Non-stop was eliminated using the MyoIA-Gal4/Gal80ts system for forty-eight hours ectopically express PCNA (purple), but not in cells that co-express UAS-LamC. Example of two cells is shown; the purple and red arrows point to the cells shown in the inset; The left cell exhibits high level of LamC (red) and low level of PCNA (purple), and the right cell exhibit low level of LamC and high level of PCNA.

Figure 5; Figure supplemental 2: Analysis of changes in chromatin actability upon loss of Not. (A) Whole genome changes in chromatin accessibility unveiled by ATAC seq divided to clusters by location along gene regions and GO ontology of each cluster. (B) MEME analysis of cluster -enrichment in DNA binding sequences associated with the indicated TFs. (C) List of all genes in the vicinity of regions that exhibit increased accessibility upon loss of Non -stop in ECs.

Figure 6; Not maintains the protein level and intranuclear localization the NIC subunits, (A-H) Representative confocal images of the midgut tissue using the indicated antibodies (red) and expressing the indicated transgenes in EC using the MyoIA-Gal4/Gal80ts system. UASLacZ (A, C, E, G), UAS-Not RNAi (B, D, F, H). DAPI marks DNA (blue), and scale bar is $10 \mu \mathrm{M}$. White arrows points to cells shown in insets, and scale bar is $10 \mu \mathrm{M}$. (I-L) Quantification of 3 biological experiments is shown (M-T) Non-stop regulate large-scale organization of the 
nucleus. Representative confocal images of the midgut tissue using the indicated antibodies (red) and expressing the indicated transgenes in EC using the MyoIA-Gal4/Gal80ts system. UAS-LacZ (M-P), UAS-Not RNAi (Q-T).

Figure 6; Supplemental Figure 1: NIC subunits are required for maintaining large-scale organization of the EC nucleus. (A-O) Confocal images of the midgut tissue using the indicated antibodies (red) and expressing the indicated transgenes in EC using the MyoIAGal4/Gal80ts system, DAPI marks DNA (blue) and scale bar is $10 \mu \mathrm{M}$ M (A, D, G, J, M ) Mtor; (B, E, H, K, N) Coilin (C, F, I, L, O) Nop60B ; (A-C) Control, (D-F) UAS-Cp190 RNAi; (GI) UAS-e(y)2 RNAi (J-L) Mod(Mdg4) RNAi (M-O) Nup98-96 RNAi.

Figure 6; Supplemental source data: Quantification of cell populations described in 6I-L.

Figure 7: The protein levels of the Not-CP190 complex subunits decline upon aging and is restored upon continues expression of Not in aged ECs. (A-O) Representative confocal images of the midgut tissue using the indicated antibodies (red) and expressing the indicated transgenes in EC using the MyoIA-Gal4/Gal80ts system. (A-E) Young 4 days old guts, (F-J) Five weeks old guts expressing UAS-lacZ. (K-O) Five weeks old guts expressing UAS-Not. DAPI marks DNA (blue), and scale bar is $10 \mu \mathrm{M}$. (P-T) Quantification of similar experiments presented in A-O. $* * * *=P<0.0001, * * * P<0.001 ; * * P<0.01 ; *=P<0.1$

Figure 7; source data: Quantification of cell populations described in 7P-T.

Figure 8: Expression of Non-stop restore large-scale organization of aged ECs: (A-O) 
Confocal images of the midgut tissue using the indicated antibodies and expressing the indicated transgenes in EC using the MyoIA-Gal4/Gal80ts. DAPI marks DNA, and scale bar is

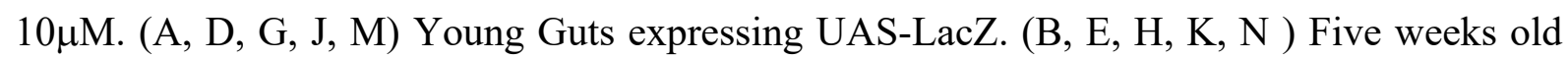
guts expressing control (UAS-GFP). (C, F, I, L, O) Five weeks old guts expressing UAS-Nonstop. (A-C) $\alpha$-LamC; (D-F) Mtor; (G-I) $\alpha$-Histone H; (J-L) $\alpha$-Lam1Dm0; (M-O) $\alpha$-Nop60B..

\section{Figure 8 Supplemental Figure 1: Expression of Non-stop restore large-scale organization} of aged ECs: (A-H) Confocal images of the midgut tissue using the indicated antibodies and expressing the indicated transgenes in EC using the MyoIA-Gal4/Gal80ts (A, D, G) Young Guts expressing the indicated UAS-LacZ. Five weeks old guts expressing the indicated control . (C, F, H) Five weeks old guts expressing UAS-Non-stop. (A-C) $\alpha$-Coilin; (D-F) $\alpha-H P 1 b$; (GI) $\alpha$-Otefin.

Figure 9: Continues Expression of Not in ECs suppresses aging phenotypes in the midgut.

(A-N) Confocal images of the midgut tissue using the indicated antibodies and expressing the indicated transgenes in EC using the MyoIA-Gal4/Gal80ts. DAPI marks DNA and scale bar is $10 \mu \mathrm{M}$. (A, E, I, L) midguts derived from 2-4 days old flies (young) (B, F, J, M) Midguts derived from 5 weeks old flies expressing control (UAS-LacZ). (C, G, K, N) Midguts derived from 5 weeks old flies expressing UAS-Not. DAPI marks DNA (blue), and scale bar is $10 \mu$ M.Arm, Armadillo; SSK Snakeskin. (D, H) Quantification of similar experiments shown in $(\mathrm{A}-\mathrm{C}) .{ }^{* * * *}=P<0.0001, * * * P<0.001 ; * * P<0.01 ; *=P<0.1$ (O-P) Aging impairs gut integrity as evident by the leakage of blue-colored food into the abdomen (smurf assay). Continues expression of Not but not control using the MyoIA-Gal4/Gal80ts for five weeks safeguards gut integrity, $\mathrm{n}=48,38$ respectively; $P<0.001$, 
Figure 9; Source data: Quantification of cell populations described in 8J-L.

\section{Supplemental Tables:}

\section{Key Resources table}

Table S1: Results of primary and secondary transgenic RNAi screens

Table S2: Proteomic analysis of Non-stop bound proteins

Table S3: RNA-seq of Not-regulated genes

Table S4: ATAC-seq profiling of non-stop dependent changes in chromatin accessibility

Table S5: Gene clustering of Non-stop closed regions (complement Fig. 5 Figure Supp. 3)

\section{Materials and Methods:}

- Key resource table with fly stocks and antibodies used in this study

- Plasmids and Primers used in this study

- Chemicals used

Methods:

- In vitro binding

- Direct Yeast 2 Hybrid

- Proteomic analysis of Non-stop associated proteins

- $\quad$ RNAi in Drosophila S2 cells

- Conditional expression of transgenes in specific gut cells

- Conditional G-TRACE analysis

- Gut dissection and immunofluorescence detection

- Gut integrity and tracing of organismal survival 
- Genomic analysis; RNA-seq, ATAC-seq and bioinformatics analyses including RNA extraction, cDNA preparation and Gene expression and RNA-seq, and bioinformatics analyses.

- Statistical analysis

Fly stocks used in this study: Fly stocks were maintained on yeast-cornmeal-molasses-malt extract medium at $18^{\circ} \mathrm{C}$ or as stated in the text. UAS- RNAi used in the screen are described under Table S1.

UAS and Gal4 transgenic lines used: All transgenic RNAi lines used for the Ub/Ubl screen are detailed in Table S1. All other lines used in this study are described in the

Antibodies used in this study: All primary and secondary antibodies used are described in the Key resource table.

\section{Plasmids and primers:}

pRmha3 C-HAx2-FLx2-nonstop-735 - was as described in (Cloud et al. 2019).

Plasmids for in vitro binding: CP190 CT (aa, 468-1096) was PCR-amplified using primers 5'tttggtaccgggecetggetgtgectg-3' and 5'-tttctcgagtgeggecgeagatcttag-3' and subcloned into pET32a $(+)$ vector (Merck Biosciences) in frame with 6xHis tag using restriction sites KpnI and Xhol.

CP190 NT (aa, 1-524) was PCR-amplified using primers 5'- tttcatatgggtgaagtcaagtccgtg -3' and 5'- tttctcgagcatgtggaaatgcagttcceg -3 ' and subcloned into pET32a $(+)$ vector $($ Merck Biosciences) in frame with 6xHis tag using restriction sites NdeI and XhoI. E(y)2 was PCR-amplified using primers 5'- tttggatccccggaattcccgacgatgag-3' and 5'tttgcggccgcttaggattcgtcctctggc-3' and subcloned into pET32a(+) vector (Merck Biosciences) in frame with 6xHis tag using restriction sites BamHI and NotI

Plasmids used in the yeast two-hybrid assay: The full-sized Not (aa 496) was PCR-amplified using primers 5'-ttgaattcatgtccgagacgggttgtc-3' and 5'-ttgtcgacttactcgtattccagcacatt-3' and 
subcloned into pGBT9 vector (Clontech) in frame with DNA-binding domain of GAL4 using restriction sites EcoRI and SalI.

The full-sized CP190 (aa 1096) was PCR-amplified using primers 5'ttcccgggcatgggtgaagtcaagtccg-3' and 5'-tttggaggagctatatttactaagatct-3' and subcloned into pGAD424 vector (Clontech) in frame with activation domain of GAL4 using restriction sites SmaI and BamHI. Fragments of CP190 from first to fourth zinc fingers was PCR-amplified using primers 5 '-ttgaattcgagaatactactgggecct-3' and 5'-ttgtcgacgecatcctccaaagcctg-3', from second to third - 5'-ttgaattcgegctttgtgagcattgc-3' and 5'-ttgtcgacgttgtcgtccgtgtgcac-3' and then subcloned into pGAD424 vector (Clontech) in frame with activation domain of GAL4 using restriction sites EcoRl and Sall.

Corresponding primers were used to make full-sized deletion variants of CP190:

CP19044 5'-aaggtaccggagcaggctttgga-3' and 5'-aaggtacccactgctgcttgttgtcg-3';

CP190வ3-4 5'-aaggtaccggagcaggctttgga and 5'-aaggtaccaacgtatacagcagcgac-3';

CP190வ2-4 5'-aaggtaccggagcaggctttgga and 5'-aaggtacccgcgccggatcaattg-3';

CP19041-4 5'-gcctggctgaaggagcaggctttggagga and 5'-cctgctccttcagccagggccagtagtat-3', Primers used for Non-stop RNAi in Drosophila cells

- Not-RNAi forward - 5'-cggaattccgaattaatacgactcactatagggatttaatctggaaccatgcgaa-3'

- Not-RNAi reverse - 5'-cggaattccgaattaatacgactcactatagggaaatgtcccaaaacggatcgta-3'

Chemicals: Bromophenol Blue (Sigma \#B5525), Guanidine hydrochloride (Sigma \#G4505), NP40 (Igepal CA-630) (Sigma \#I3021), Triton X-100 (Amresco \#0694), Acrylamide (BisAcrylamide 29:1) (Biological Industries \#01-874-1A), Ammonium Persulfate (Sigma \#A9164), TEMED (Sigma \#T-7024), L-Glutamine (Gibco \#25030024), MG132 (Boston Biochemicals), Blot Qualified BSA (Biological Industries \#PRW3841), Agarose (SeaKem ${ }^{\circledR}$ LE Agarose- Cambrex Bio Science \#CAM-50004), Bradford Protein Assay (BioRad \#500- 
0006), EZ-ECL (Biological Industries \#20-500-500), FD\&C blue dye \#1, Cyclohexamide (Sigma \#01810).

\section{Methods:}

In vitro binding: 6xHis-tagged proteins were xpressed and purification from E. coli BL-21 (DE3), using Ni-NTA agarose beads. His-tagged protein were induced with $0.5 \mathrm{mM}$ IPTG for $5 \mathrm{~h}$ at $30^{\circ} \mathrm{C}$ and subsequently immobilized on with Ni-NTA agarose beads. Nuclear extract derived from Non-stop expressing S2 cells was prepared similar to the described in (Cloud et al, 2019), see "Non-denaturing extract": Stably transfected cells were resuspended in Extraction Buffer (20 mM HEPES (pH7.5), 25\% Glycerol, $420 \mathrm{mM} \mathrm{NaCl}, 1.5 \mathrm{mM} \mathrm{MgCl}_{2}$, $0.2 \mathrm{mM}$ EDTA, 1:100 ethidium bromide with protease inhibitors added. 1\% NP-40 was added and the cells were pipetted up and down until the solution was homogenous. They were placed on ice for one hour with agitation every 10-15 minutes. They were then centrifuged for 30 minutes at $4{ }^{\circ} \mathrm{C}$ at $20,000 \times \mathrm{g}$. An equal volume of Dignum A buffer (10 mM HEPES (pH 7.5), $1.5 \mathrm{mM} \mathrm{MgCl} 2,10 \mathrm{mM} \mathrm{KCl}$ ) was added to the lysates in order to adjust the salt concentration to $210 \mathrm{mM} \mathrm{NaCl}$.

Binding was performed using $0.5 \mathrm{mg}$ of S2 cell extract expressing HF-Non-stop and the indicated His-tagged proteins immobilized to Ni-NTA beads using binding buffer $(20 \mathrm{mM}$ Hepes-KOH pH 7.7, 150mM NaCl, $10 \mathrm{mM} \mathrm{MgCl} 20.1 \% \mathrm{mM} \mathrm{ZnCl}_{2}, 0.1 \% \mathrm{NP} 40,10 \%$ Glycerol and protease inhibitors) for over-night in rotation. Subsequently, beads were collected and washed four times with wash buffer, and proteins resolved over SDS-PAGE and detected by western blot analysis.

Yeast two-hybrid assay (Y2H): Y2H was carried out using yeast strain pJ69-4A (MATa trp1-901 leu2-3,112 ura3-52 his3-200 gal4 $\Delta$ gal80 $\Delta$ GAL2-ADE2 LYS2::GAL1-HIS3 met2::GAL7-lacZ), with plasmids according to Clontech protocols . In brief, for growth 
assays, AD (activation domain of GAL4) - and BD (DNA-binding domain of GAL4) -fused plasmids were co-transformed into yeast strain pJ69-4A by the lithium acetate method, as described by the manufacturer with some modifications. Transformed cells were plated on selective medium lacking Leu (leucine biosynthesis gene Leu2 is expressed from pGAD424 plasmid) and Trp (tryptophan biosynthesis gene Trp1 is expressed from pGBT9 plasmid) ('medium-2'). The plates were incubated at $30^{\circ} \mathrm{C}$ for 2-3 days. Afterward, the colonies were streaked out on plates on selective medium lacking either Leu, Trp and His (histidine biosynthesis gene His3 is used as reporter in $\mathrm{Y} 2 \mathrm{H}$ assay) ('medium-3'). The plates were incubated at $30^{\circ} \mathrm{C}$ for 3-4 days, and growth was assessed. The positive growth of yeast on selective 'medium-3' indicates a physical interaction between protein molecules fused with $\mathrm{AD}$ and $\mathrm{BD}$. Each assay was prepared as three independent biological replicates with three technical repeats.

Proteomic analysis: Multidimensional protein identification technology and Mass spectrometry data processing were identical to the described in detail at (Cloud et al. 2019); Multidimensional protein identification technology (MudPIT) and Mass spectrometry data processing were identical to that described in Cloud et al. 2019. MudPIT: TCA-precipitated protein pellets were solubilized using Tris- $\mathrm{HCl} \mathrm{pH} 8.5$ and $8 \mathrm{M}$ urea, followed by addition of TCEP (Tris(2-carboxyethyl)phosphine hydrochloride; Pierce) and CAM (chloroacetamide; Sigma) were added to a final concentration of $5 \mathrm{mM}$ and $10 \mathrm{mM}$, respectively. Proteins were digested using Endoproteinase Lys-C at 1:100 w/w (Roche) at $37^{\circ} \mathrm{C}$ overnight. Samples were brought to a final concentration of $2 \mathrm{M}$ urea and $2 \mathrm{mM} \mathrm{CaCl}_{2}$ and a second digestion was performed overnight at $37^{\circ} \mathrm{C}$ using trypsin (Roche) at 1:100 w/w. The reactions were stopped using formic acid (5\% final). The digested size exclusion eluates were loaded on a split-triplephase fused-silica micro-capillary column and placed in-line with a linear ion trap mass 
spectrometer (LTQ, Thermo Scientific), coupled with a Quaternary Agilent 1100 Series HPLC system. The digested Non-stop and control FLAG-IP eluates were analyzed on an LTQOrbitrap (Thermo) coupled to an Eksigent NanoLC-2D. In both cases, a fully automated 10step chromatography run was carried out. Each full MS scan $(400-1600 \mathrm{~m} / \mathrm{z})$ was followed by five data-dependent MS/MS scans. The number of the micro scans was set to 1 both for MS and MS/MS. The settings were as follows: repeat count 2; repeat duration $30 \mathrm{~s}$; exclusion list size 500 and exclusion duration 120 s, while the minimum signal threshold was set to 100 . Mass Spectrometry Data Processing: The MS/MS data set was searched using ProLuCID (v. 1.3.3) against a database consisting of the long (703 amino acids) isoform of non-stop, 22,006 nonredundant Drosophila melanogaster proteins (merged and deduplicated entries from GenBank release 6, FlyBase release 6.2,2 and NCI RefSeq release 88), 225 usual contaminants, and, to estimate false discovery rates (FDRs), 22,007 randomized amino acid sequences derived from each NR protein entry. To account for alkylation by CAM, 57 Da were added statically to the cysteine residues. To account for the oxidation of methionine to methionine sulfoxide, $16 \mathrm{Da}$ were added as a differential modification to the methionine residue. Peptide/spectrum matches were sorted and selected to an FDR less than 5\% at the peptide and protein levels, using DTASelect in combination with swallow, an in-house software.

The permanent URL to the dataset is: ftp://massive.ucsd.edu/MSV000082625. The data is also $\begin{array}{lll}\text { accessible from: } & \text { ProteomeXChange } & \text { accession: }\end{array}$ http://proteomecentral.proteomexchange.org/cgi/GetDataset?ID=PXD010462. MassIVE Accession ID: MSV000082625 - ProteomeXchange | Accession ID: PXD010462.

RNAi in S2 cells: S2 Schneider DRSC cells (Drosophila Genomics Resource Center \#181, RRID:CVCL_Z992) were maintained in Schneider's media supplemented with 10\% fetal bovine serum and 1\% penicillin-streptomycin (Thermo-Fisher, Catalog number: 15070063 , $5000 \mathrm{U} / \mathrm{ml}$ ) RNAi in S2 cells was performed as described in (Abed et al. 2011). 
Conditional expression of transgenes in specific gut cells: Conditional expression of transgenic lines in specific midgut cells was achieved by activating a UAS-transgene under the expression of the cell-specific Gal4-drivers together with the tubGal80 ${ }^{\text {ts }}$ construct (Jiang et al., 2009). Flies were raised at $18^{\circ} \mathrm{C} \cdot 2-4$ days old, F1 adult progeny were transferred to the restrictive temperature $29^{\circ} \mathrm{C}$ (Gal80 off, Gal4 on) for two days unless indicated otherwise, dissected and analyzed. At least three biological independent repeats were performed for each experiment. Where possible, multiple RNAi lines were used.

Conditional G-TRACE analysis: G-TRACE analyses was as described in (Flint-Brodsly et al. 2019) using Myo-Gal4; G-TRACE flies were crossed to UAS-LacZ; Gal80 ${ }^{\text {ts }}$ (control) or UASNon-stop RNAi ; Gal80 $0^{\text {ts }}$ and the appropriate genotypes were raised at $18^{\circ} \mathrm{C}$ (a temperature where no G-TRACE signal was detected). At 2-4-days, adult females were transferred to $29^{\circ} \mathrm{C}$ and linage tracing was performed.

Gut dissection and immunofluorescence detection: Gut fixation and staining were carried out as previously described (Shaw et al., 2010; Flint-Brodsly et al. 2019).

Gut integrity and animal survival: Young female flies from the indicated genotype were collected into a fresh vial (10 flies per vial), that were kept in a humidified, temperaturecontrolled incubator at $29^{\circ} \mathrm{C}$ for the indicated time period. Smurf assay was performed as described in (Flint-Brodsly et al. 2019). Flies were transferred into vials containing fresh food every two days and were scored for viability at the indicated time points. LT50 (lethal time in days at which $50 \%$ of the flies died) Statistical analysis was calculated using the GraphPad Prism 5.00 (GraphPad Software, San Diego, CA, USA).

\section{Genomic studies:}

RNA-sequencing: RNA Sample Preparation: RNA-seq was performed similar to the described in (Flint-Brodsly et al 2019). In brief, Adult Drosophila (2-4 days old) females, from 4 
biological repeats, in which UAS-Non-stop RNAi or control UAS-GFP RNAi were expressed in ECs using MyoIAts and dissected in Ringer's solution on ice. The solution was then discarded, and the guts were disrupted by adding $350 \mu 1 \mathrm{RLT}+\beta \mathrm{ME}$ buffer $(350 \mu 1 \mathrm{RLT}+3.5 \mu 1$ $\beta-\mathrm{ME})$. Guts were than vortex for homogenization. $350 \mu 1$ of $70 \%$ ethanol was then added and mixed well by pipetting. Guts were uploaded into RNeasy spin column and RNA purified according to the manufacture instructions. Sample quality (QC) Quality measurements for total RNA were performed using the TapeStation 2200 (Agilent).

Library preparation and data generation of RNA-sequencing: Eight RNA-seq libraries were produced using the NEBNext巴 Ultra Directional RNA Library Prep Kit for Illumina (NEB, cat no. E7420) according to manufacture protocol and starting with 100 ng of total RNA. mRNA pull-up was performed using the Magnetic Isolation Module (NEB, cat no. E7490). Two out of the twelve libraries (samples B1 \& B2) were disqualified based on low library yield and high levels of adaptor dimer. The remaining ten libraries were mixed into a single tube at an equal molar concentration. The RNA-seq data was generated on two lanes of HiSeq2500, 50SR.

NGS QC, alignment and counting 50 bp single-end reads were aligned to Drosophila reference genome and annotation file (Drosophila melanogaster BDGP6 downloaded from ENSEMBL) using TopHat (v2.0.13) allowing 2 mismatches per read with options -very-sensitive. The number of reads per gene was counted using Htseq (0.6.0).

Descriptive and RNA-seq DEGs analysis: Samples' clustering and differential expressed genes (DEGs) were calculated using Deseq2 package (version 1.10.1). The similarity between samples was evaluated using correlation matrix, shown a heat plot and Principal Component Analysis (PCA). Samples belonging to the same group were more similar then samples from different experimental groups (Figure 5 Figure Supplemental 1A). The expression $\sim 12,000$ fly transcripts were compared using DESeq2 and list of the differentially express genes (DEGs) was extracted into excel files. At adjusted p-value (p-adj) \&lt;0.01 and LogFC \&gt; 1 or LogFC \&lt; -1, 1428 DEGs were found between guts derived from the control vs Non-stop RNAi 
targeted EC. Moreover, the expression of Not was found to be downregulated by $-0.88 \log 2 \mathrm{FC}$ between Non-stop RNAi vs. Ctrl with p-adj of less than 10-9.

ATAC (Assay for Transposase-Accessible Chromatin) sequencing: Adult Drosophila (2-4 days old) females, from 3 biological repeats, in which Non-stop RNAi or GFP RNAi control were expressed in ECs using MyoIAts were dissected in ice cold Ringer's solution, and immediately placed in $25 \mu \mathrm{l}$ of ice cold ATAC lysis buffer $(10 \mathrm{mM}$ Tris-HCl, $\mathrm{pH} 7.4,10 \mathrm{mM}$ $\mathrm{NaCl}, 3 \mathrm{mM} \mathrm{MgCl} 2,0.1 \%$ IGEPAL CA-630). Lysed guts were then centrifuged at 500xg for 15 minutes at 4'C and the supernatant was discarded. The rest of the ATAC-seq protocol was performed as described in (Buenrostro et al., 2013). The final library was purified using a Qiagen MinElute kit (Qiagen) and Ampure XP beads (Ampure) (1:1.2 ratio) were used to remove remaining adapters. All samples were quantified using Qubit DNA HS assay. The final library was first checked on an Agilent Bioanalyzer 2000 for quality and the average fragment size. Successful libraries were sequenced with NextSeq 75 cycles high-output flow-cell, targeting $\sim 25$ million reads/sample.

Bioinformatic analysis of ATAC-seq: Raw reads were trimmed for adapters and aligned to the Drosophila melanogaster reference genome using bowtie2. Redundant duplicated reads that aligned to the exact locations were removed from the aligned results, and then converted to the tagAlign format with consideration of the strand shift ("+" strand reads shifted by 4bp, and "-" strand reads shifted by $-5 \mathrm{bp}$ ). The tag-Align format alignment results were used to call peaks using MAC2. Narrow peaks with $\mathrm{p}<0.1$ were reported. Peaks were compared across biological duplicates and pseudo duplicates (i.e., random subsets from a sample) to get IDR peaks that are supposed to be consensus across duplicates. Test for the difference between the two groups (control \& Noti) has been performed with the Bioconductor R package DiffBind. Significance is set by $\mathrm{FDR}<0.1$.

Comparisons between RNA and ATAC sequencing data: The two data sets of DEGs and DBAs were analyzed to create a list of significant differentially bound peaks that are close to genes 
from the top $1428 \mathrm{DE}$ genes. The thresholds that have been used to associate the peaks to genes is within $10 \mathrm{~kb}$ upstream and $10 \mathrm{~kb}$ downstream of the genes. To look for common binding motives between the two data sets, the genes were sub-divided into 4 categories: 1. peaks overlapping promoters. 2. peaks upstream of promoters. 3. peaks in genic region. 4. peaks downstream of genes. Next, I performed motif finding informatics using CONSENSUS, MDScan and MEME software.

Gene ontology analysis of mRNA expression and ATAC-seq was performed using the Database for Annotation, Visualization and Integrated Discovery (DAVID http://david.abcc.ncifcrf.gov/home.jsp; v6.7 and v6.8) using default settings (2-fold p $<0.05$ Benjamini P value for analysis E-03) (Huang et al., 2009).

Biological processes with a p-value lower than 0.05 were further analyzed with Revigo (Supek F et al., 2011). Gene ontology analyses via Cytoscape: ClueGO app (v2.2.5) in Cytoscape (v 3.4.0) was used to conduct GO enrichment analyses. In our study, ClueGO was used to identify different functional groups in the following terms: Biological Process (BP), Cellular Component (CC) and Molecular Function (MF) enrichment analysis. A p-value $\leq 0.05$ was used as the cut-off criterion.

Statistical analysis: Data was collected from three independent experiments. Statistical analysis, z-test comparisons were performed using Prism6 ANOVAs software. Significance is indicated by $* * *=\mathrm{P}<0.001$ and $* *=\mathrm{P}<0.01$. 
Figure 1 Fig. Supp. 2: Erez et al.

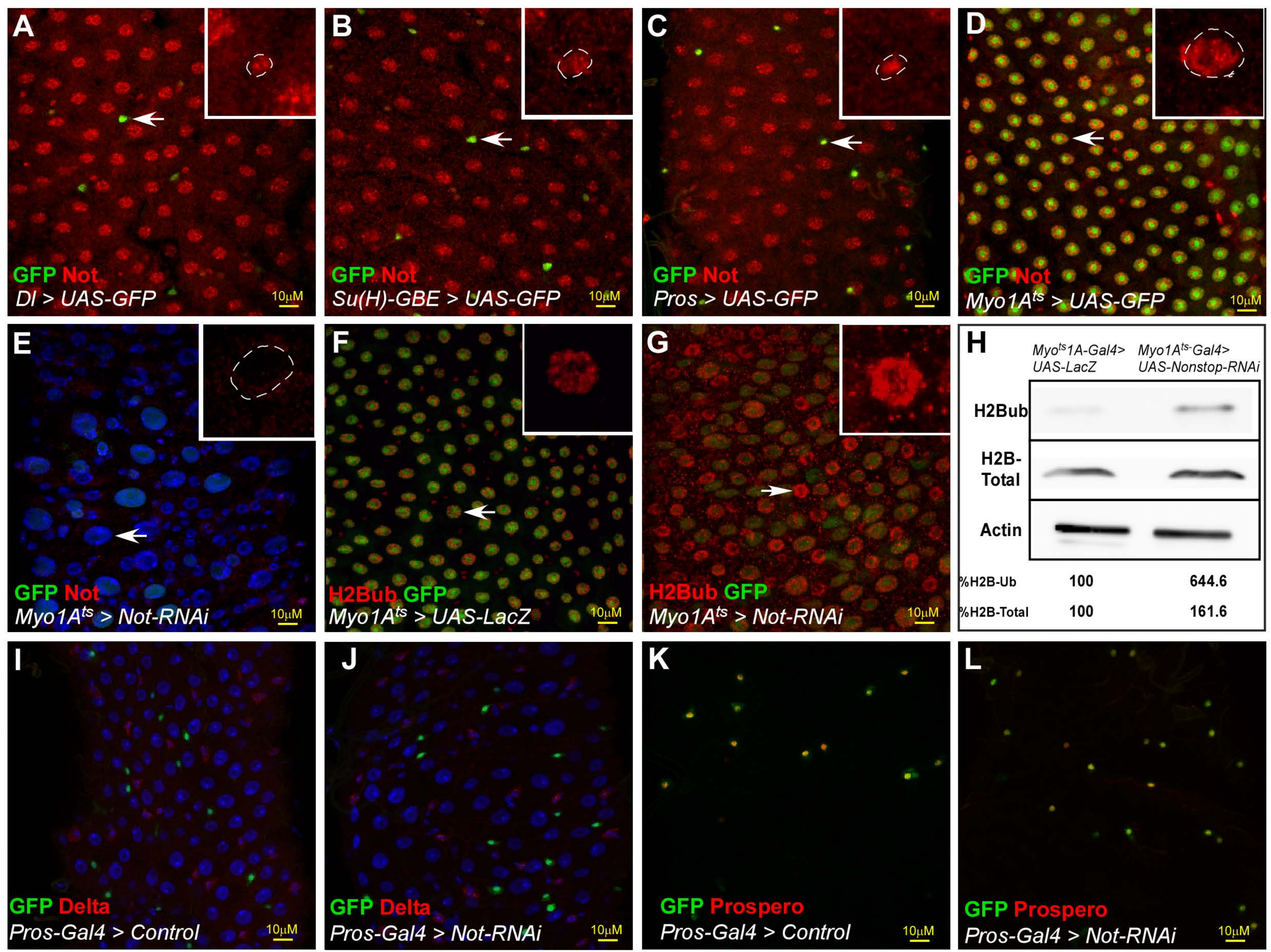


Figure 2: Erez et al.
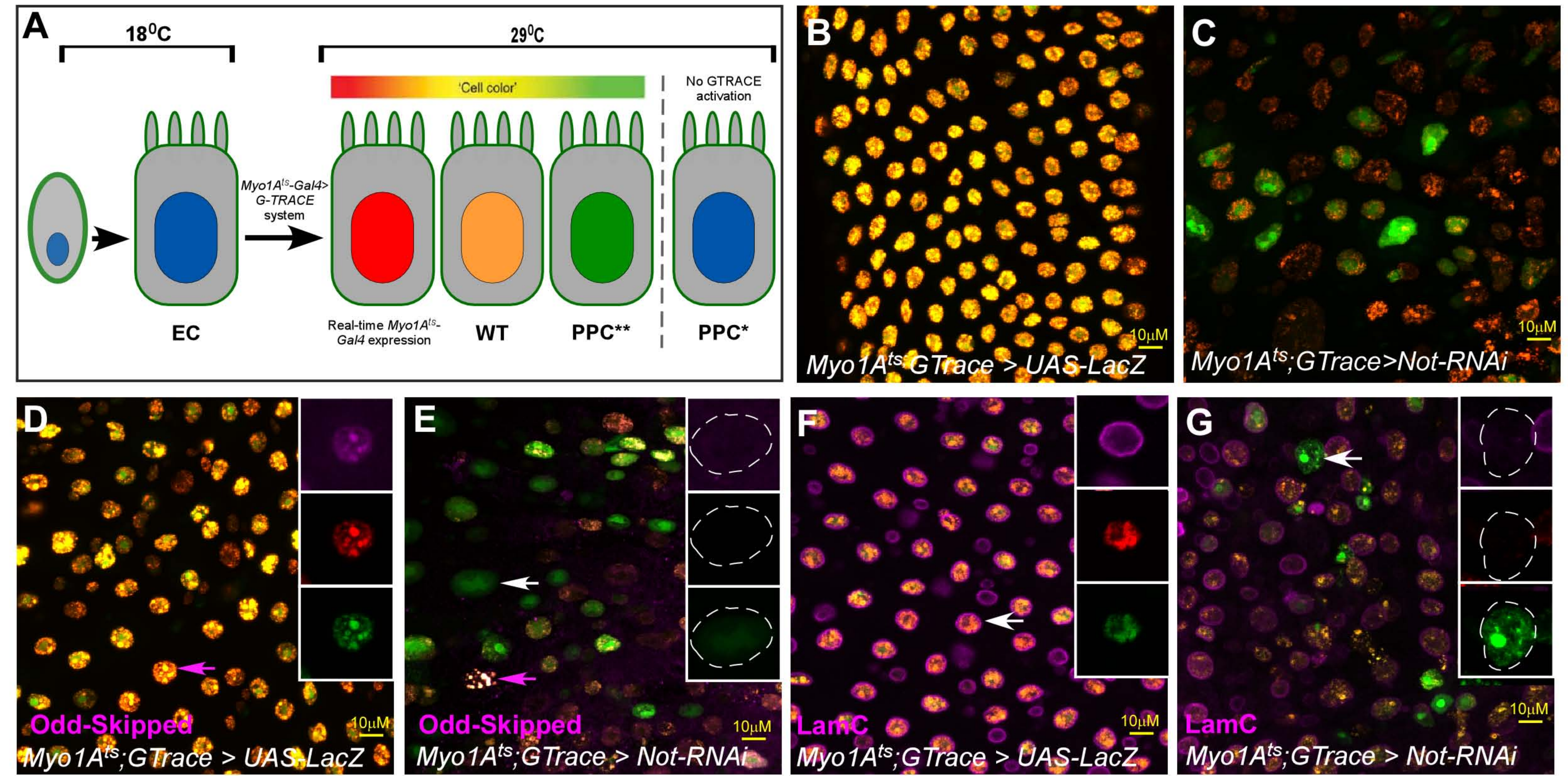

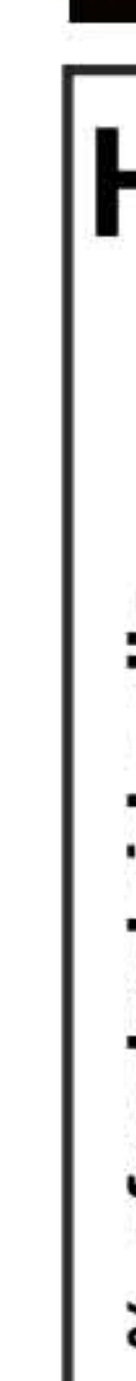
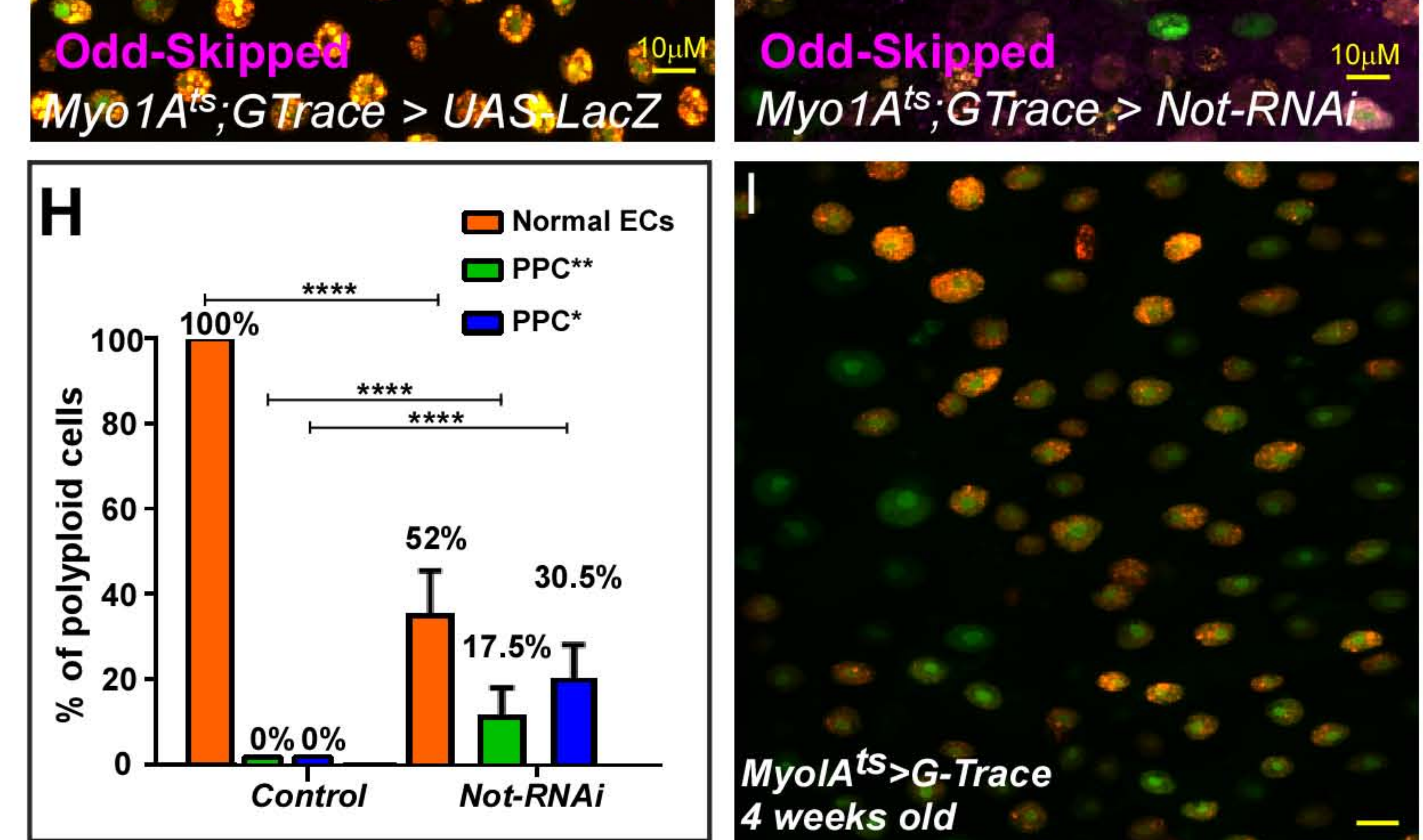

Myo1A ${ }^{\text {ts }}$; GTrace > OAS-LacZ

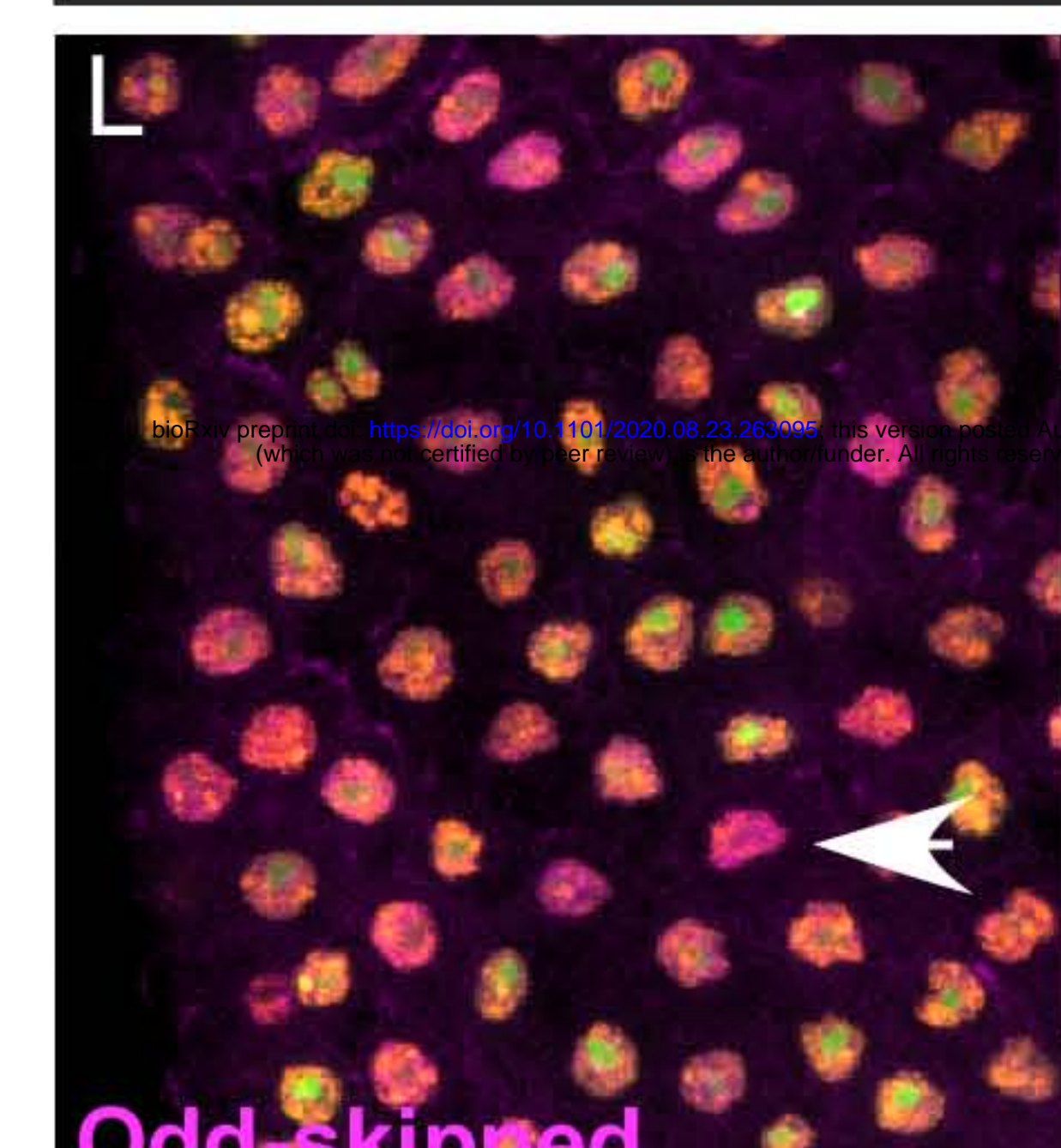

Odd 9 kipjed *

\section{MyolA ${ }^{\text {tS }}>$ G-Trace}

control 2 d

\section{4 weeks old}

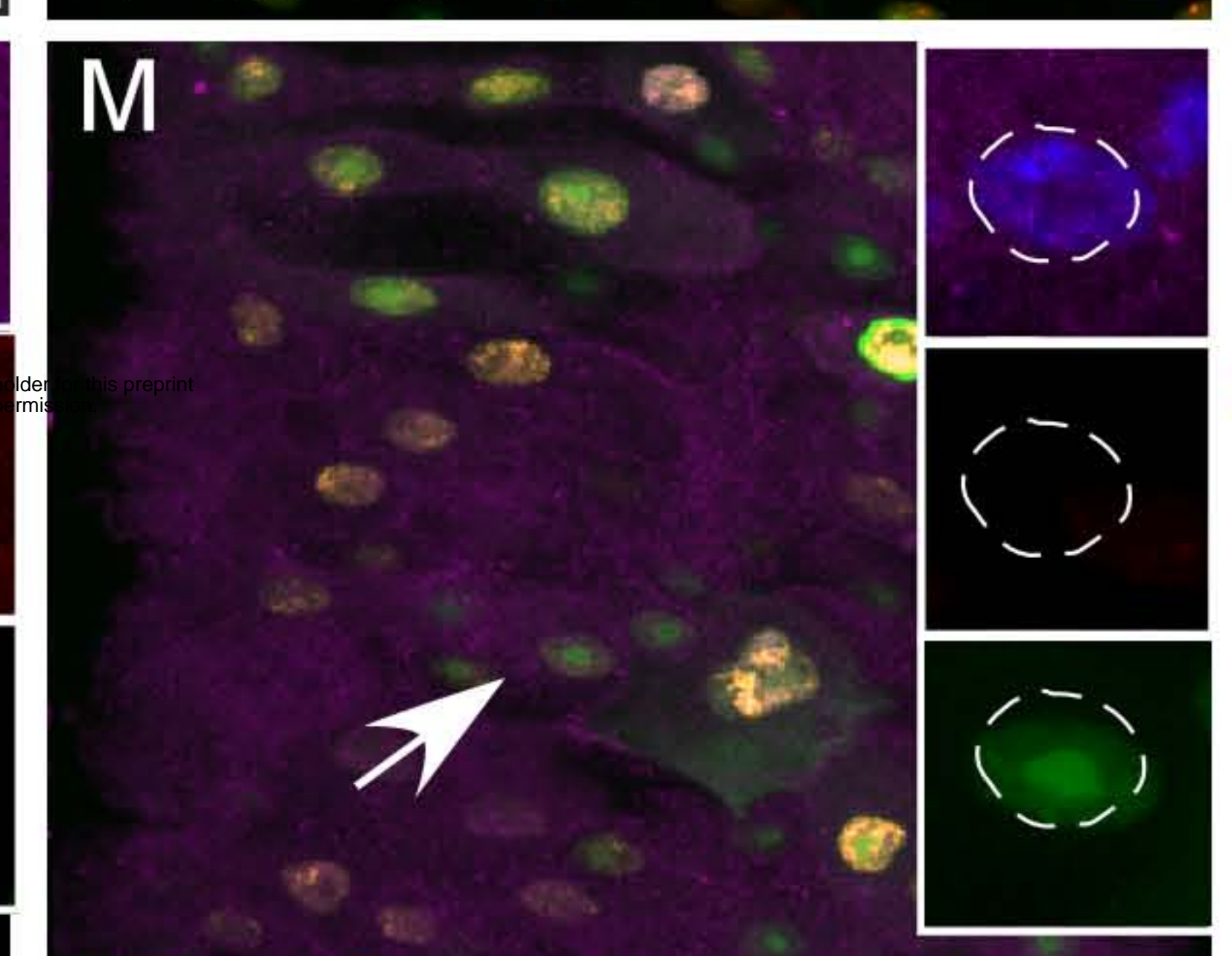

Odd-skjpped

MyolAts $>$ G-Trace

4 weeks old

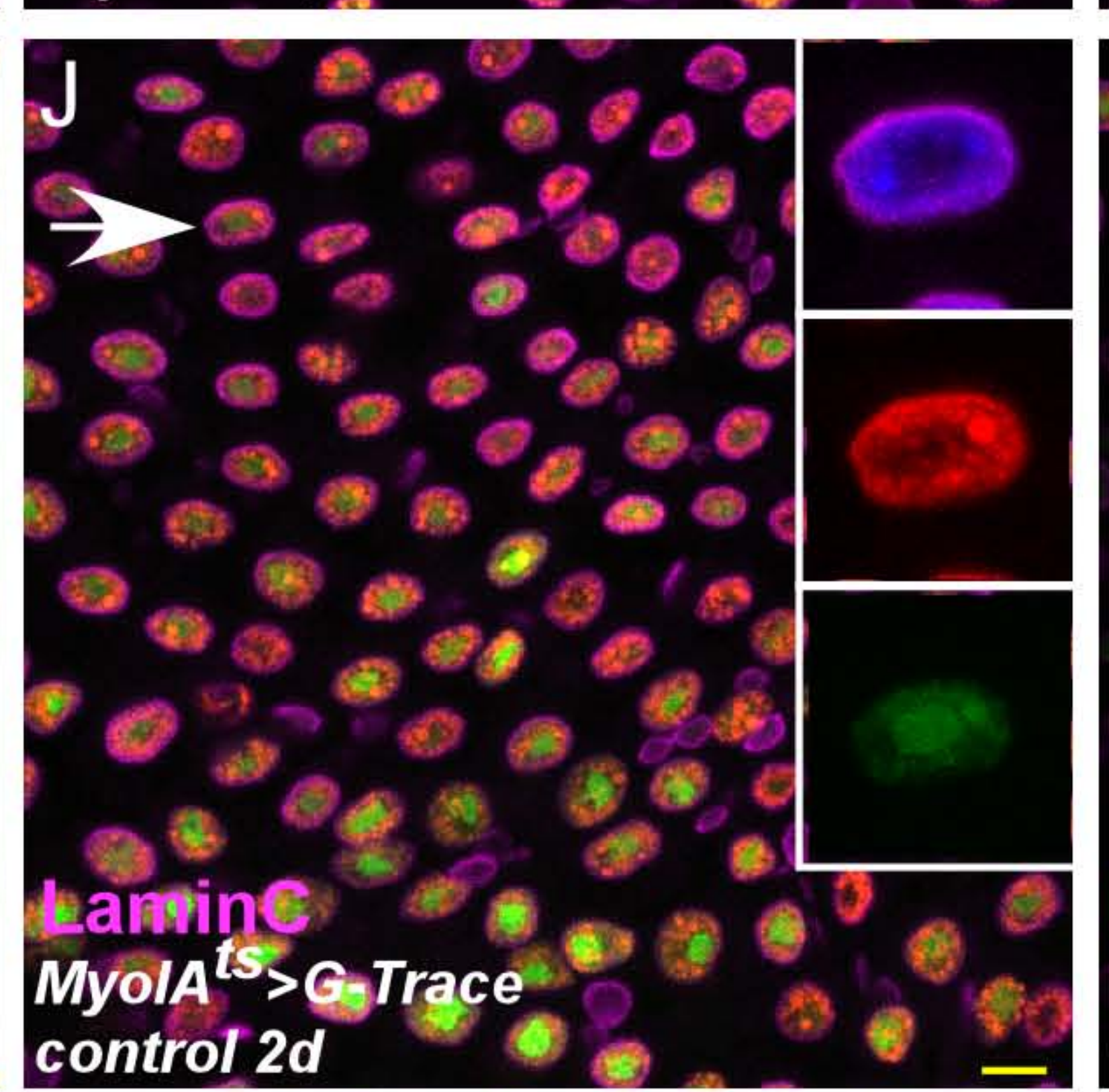

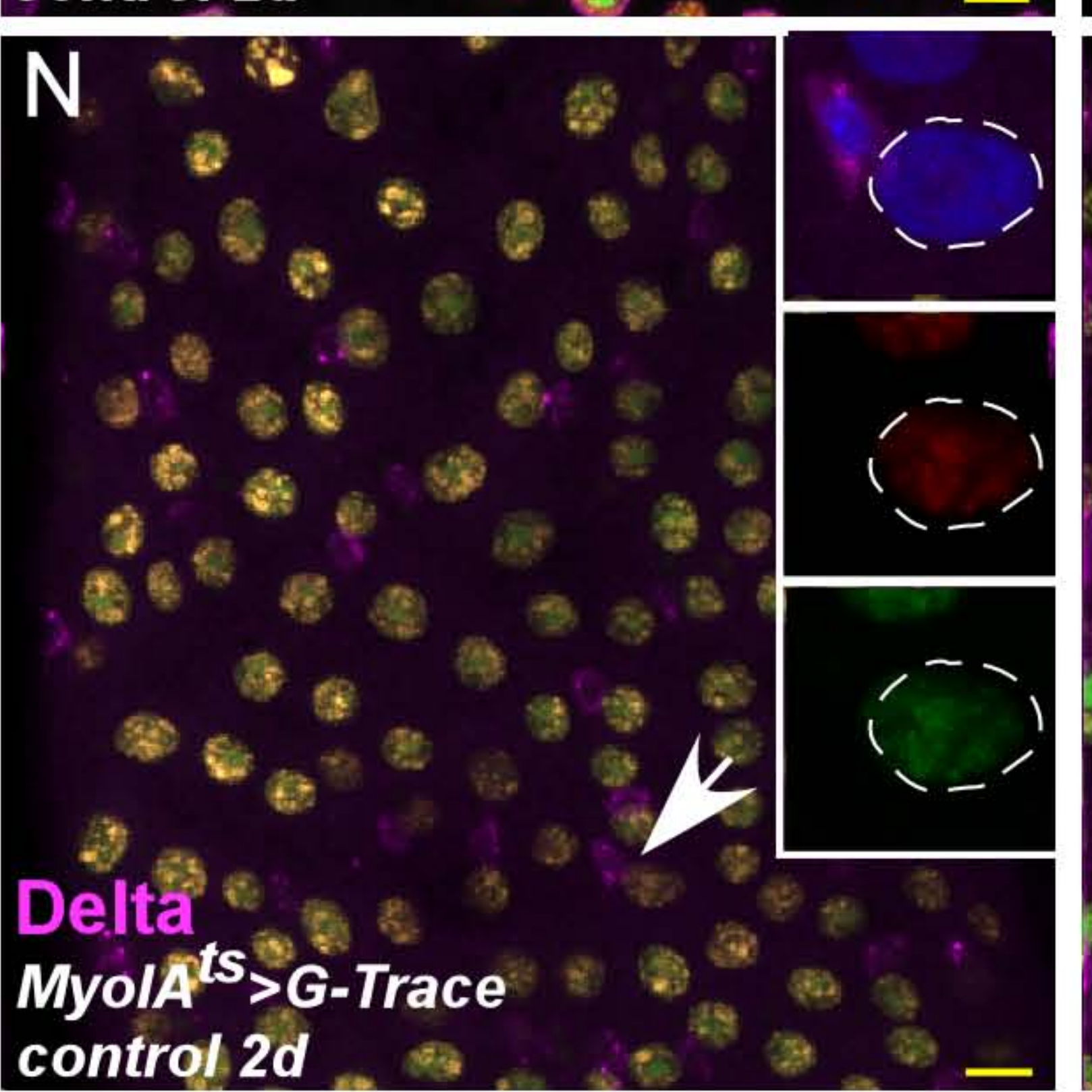

Lame

\section{Myo1 $A^{\text {ts }} ;$ GTrace $>$ Not-RNAi}

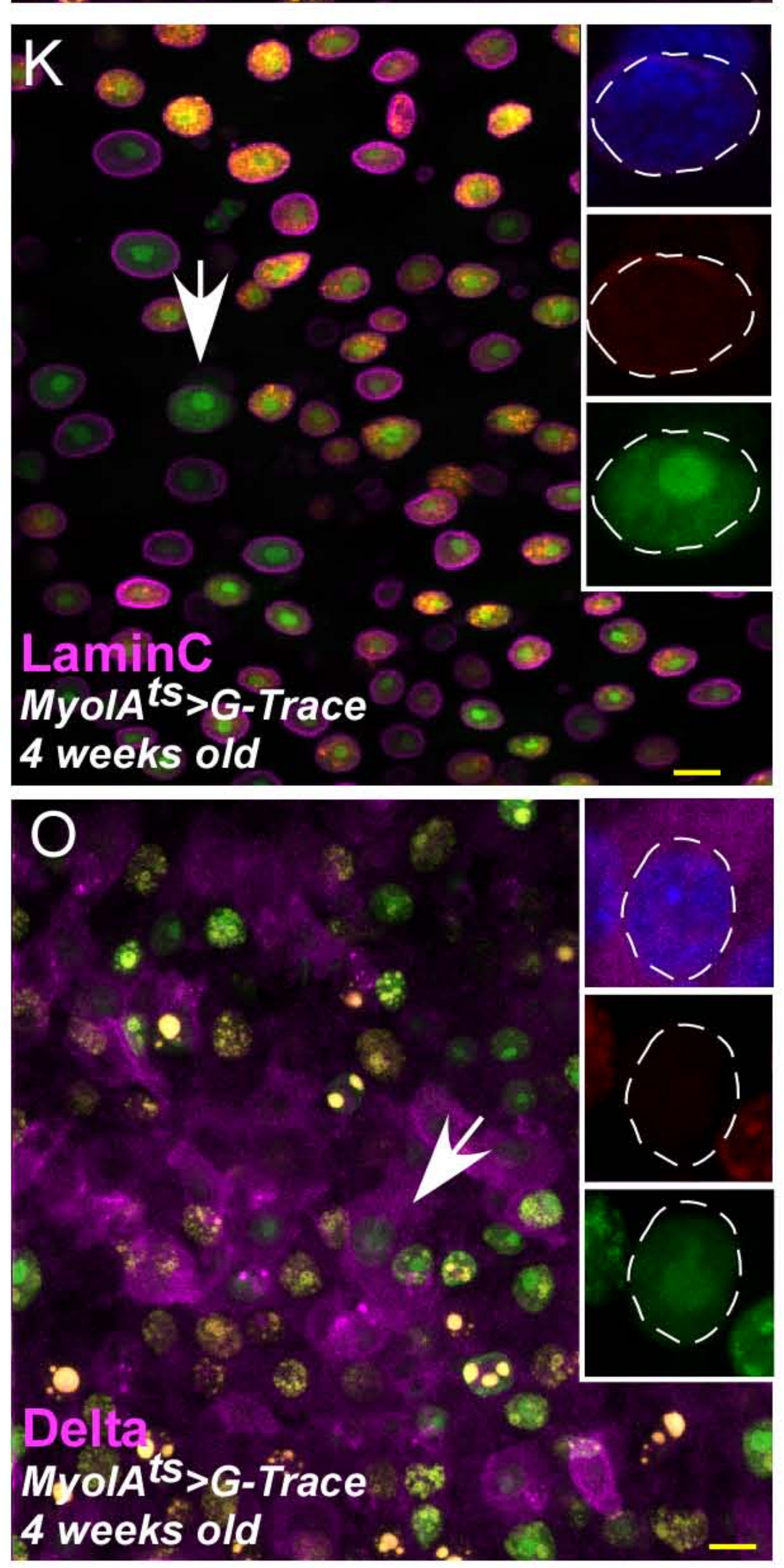

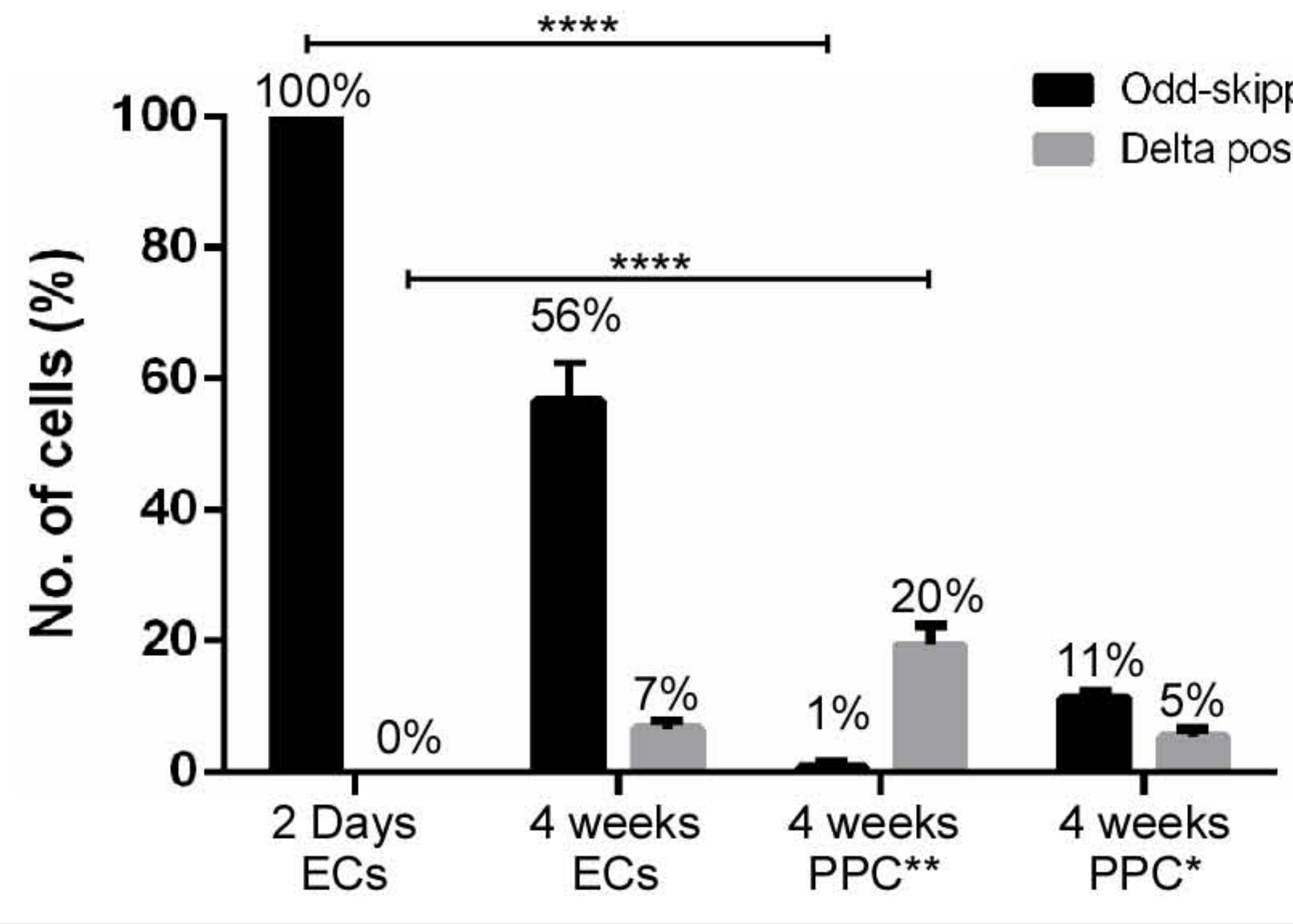


Figure 2 Fig supp. 1 : Erez et al.
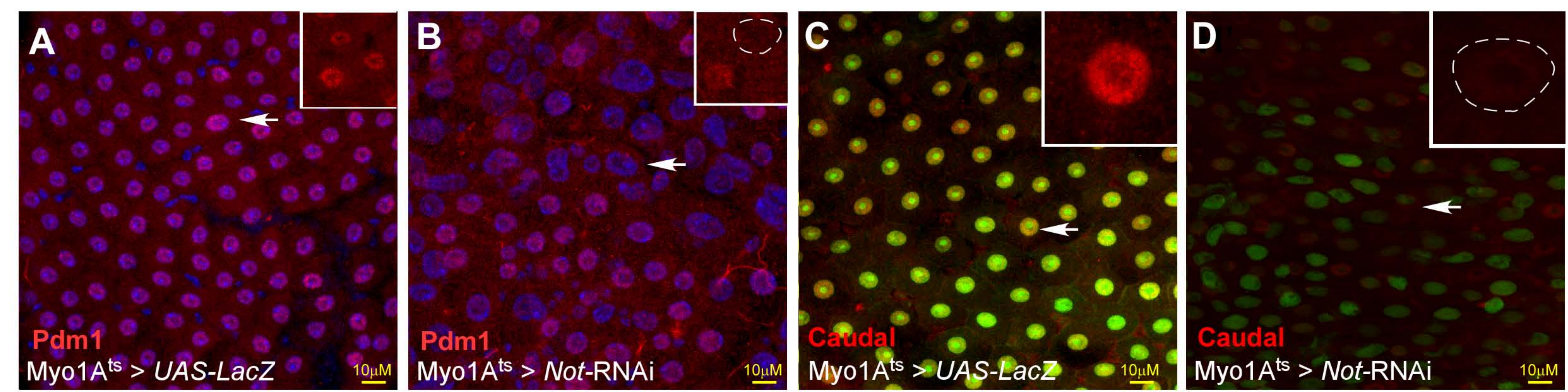
Figure 2 Fig. supp. 2: Erez et. al.




Figure 2 - Fig supp 3: Erez et. al.

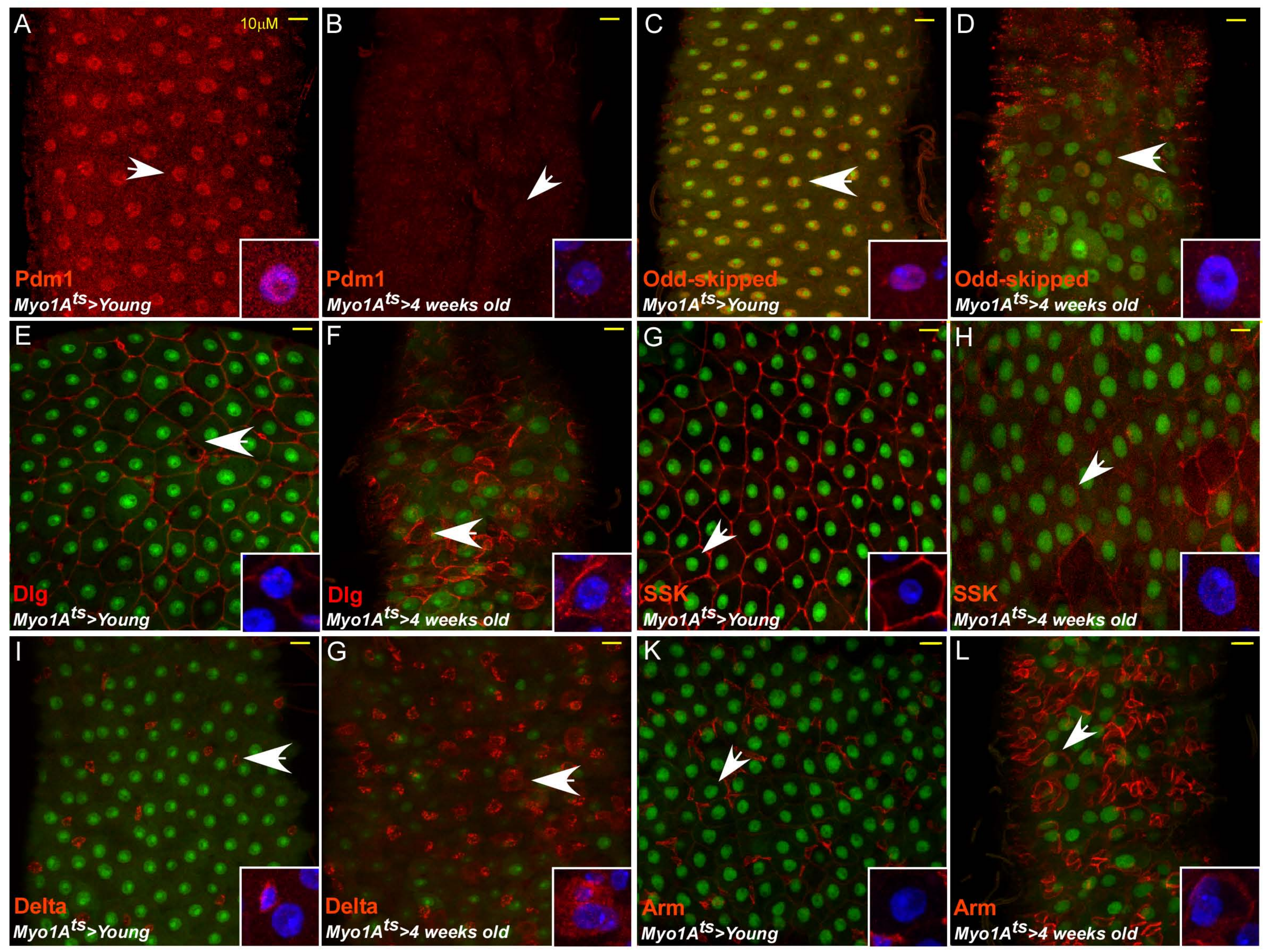


Figure 3: Erez et al

A $\begin{gathered}\text { Purify protein complexes from } \\ \text { nuclear extracts prepared from } \\ \text { S2 cells stably transfected with } \\ \text { Non-stop-FH }\end{gathered}\left|\begin{array}{c}\text { Separate purified protein } \\ \text { complexes by size using } \\ \text { size exclusion } \\ \text { chromatography }\end{array}\right| \lambda \begin{gathered}\text { Test each fraction for } \\ \text { presence of } \\ \text { deubiquitinase activity }\end{gathered} \mid \backslash \begin{gathered}\text { Identify components of } \\ \text { deubiquitinase-activity- } \\ \text { containing fractions by } \\ \text { mass spectrometry }\end{gathered}$

B



C

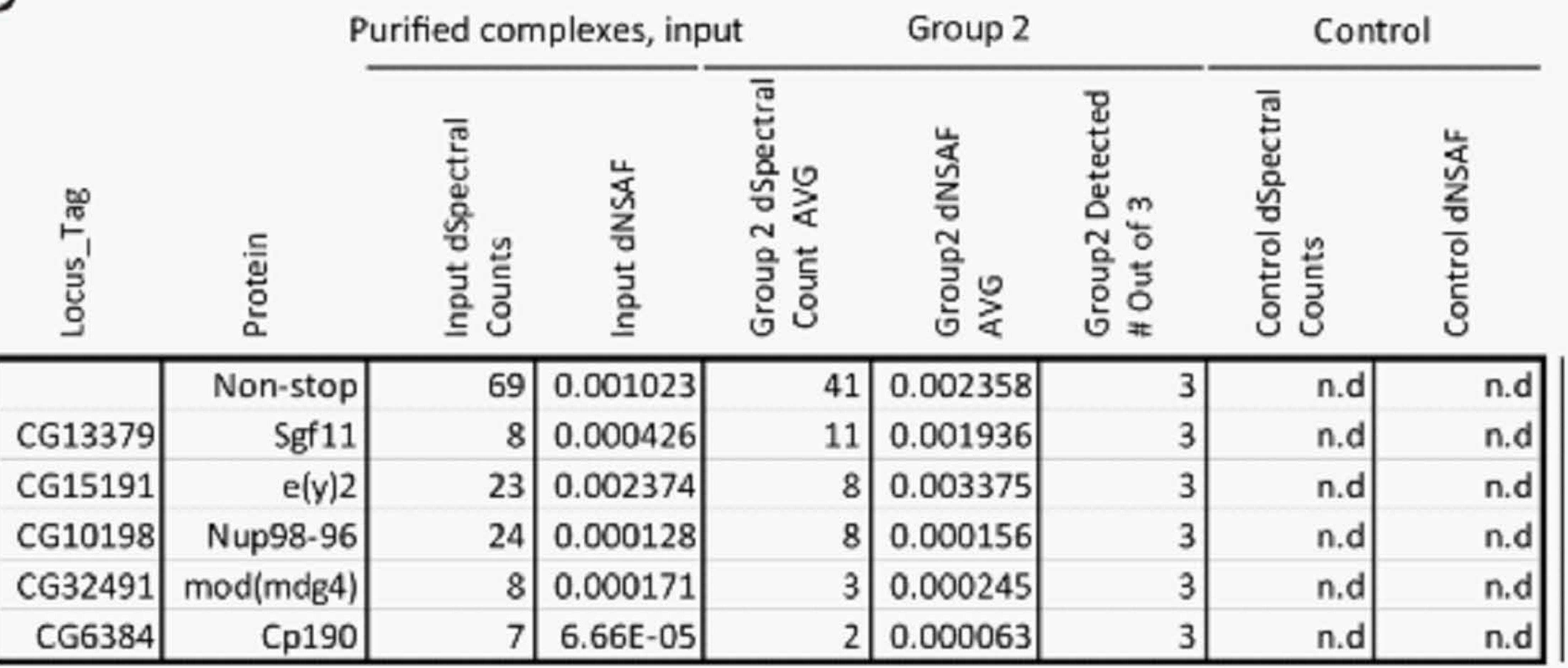

Non-stop identity complex (NIC)

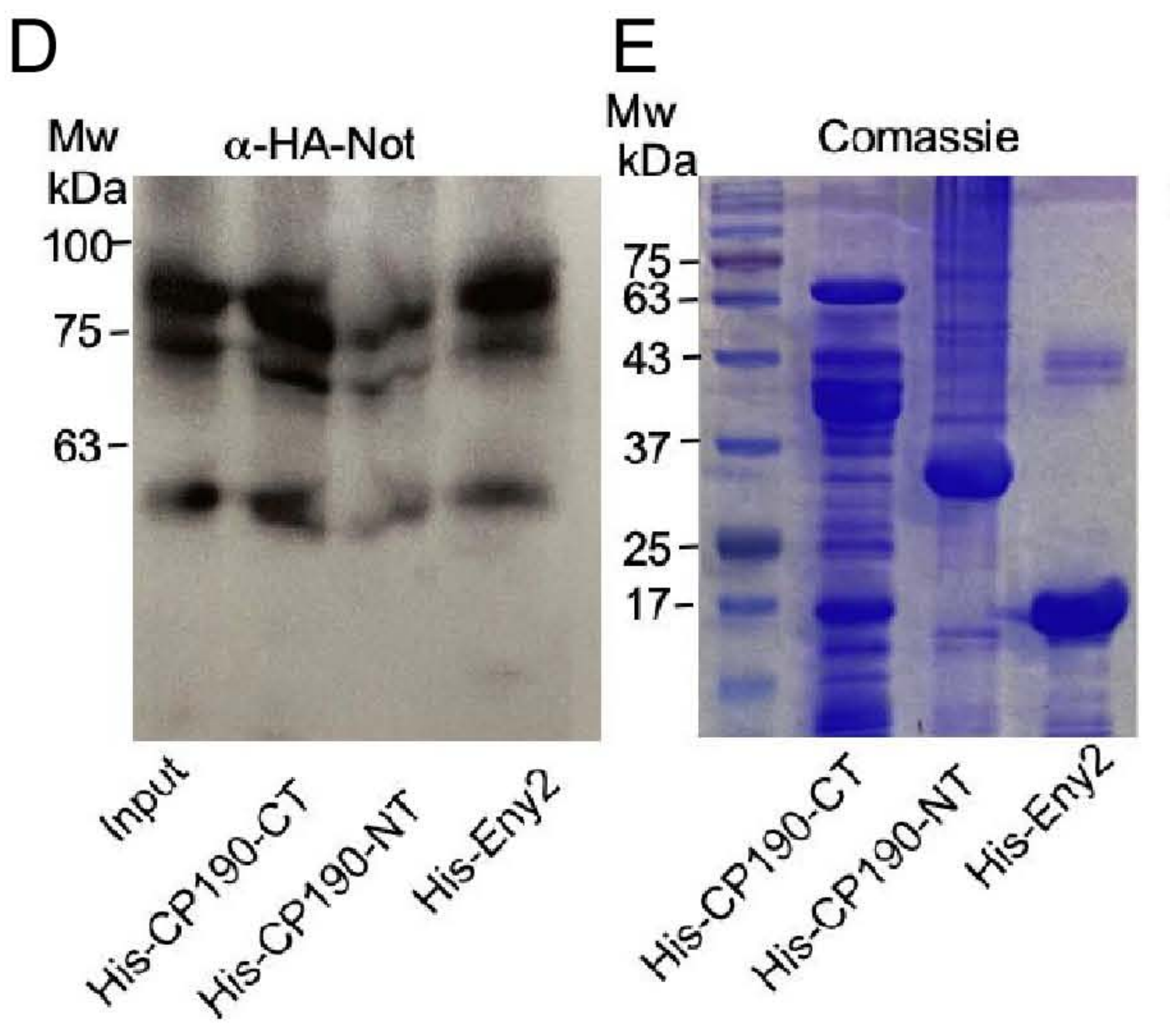

F

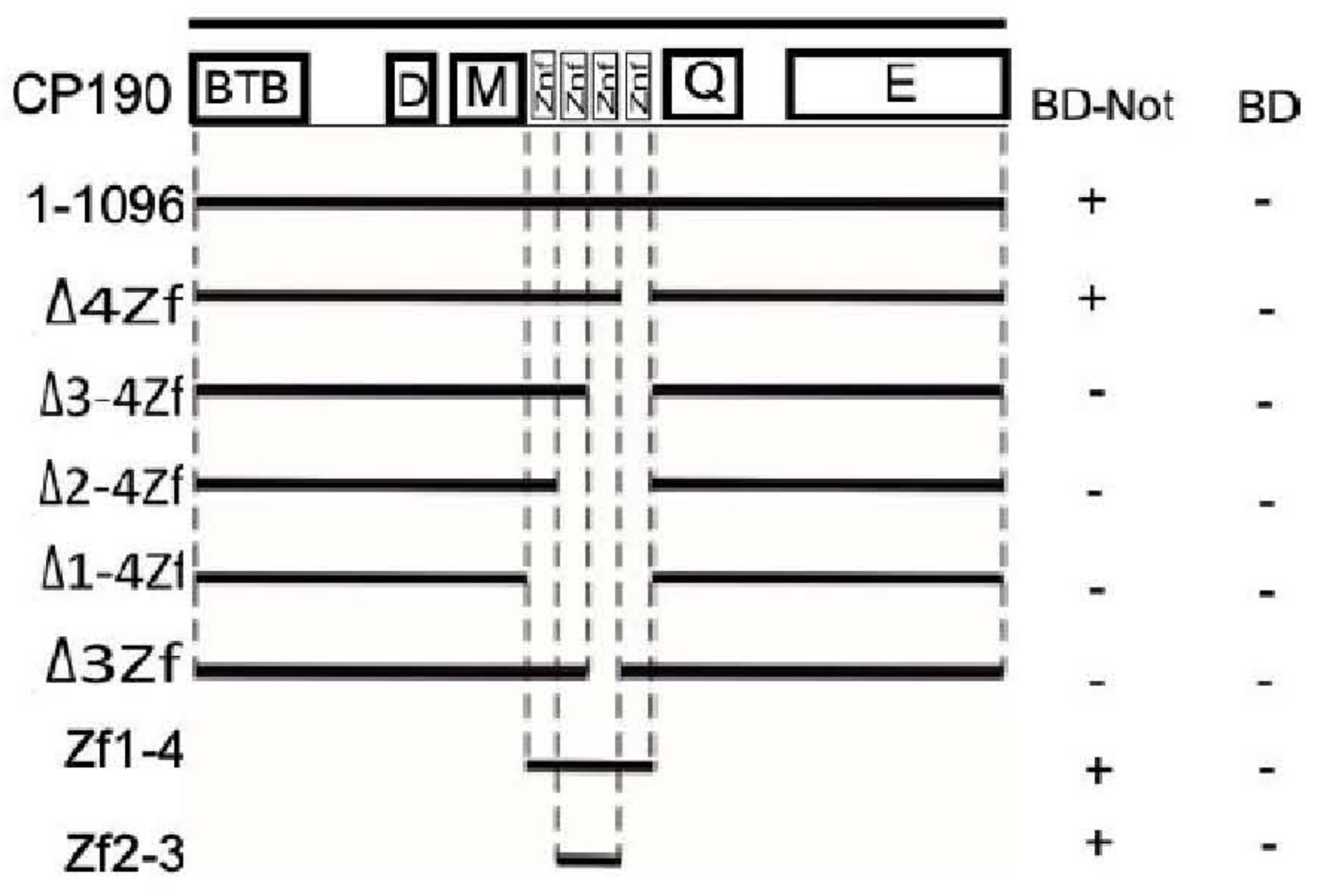


Figure 3 Fig Supp 1: Erez et al




Figure 4 Erez et al.
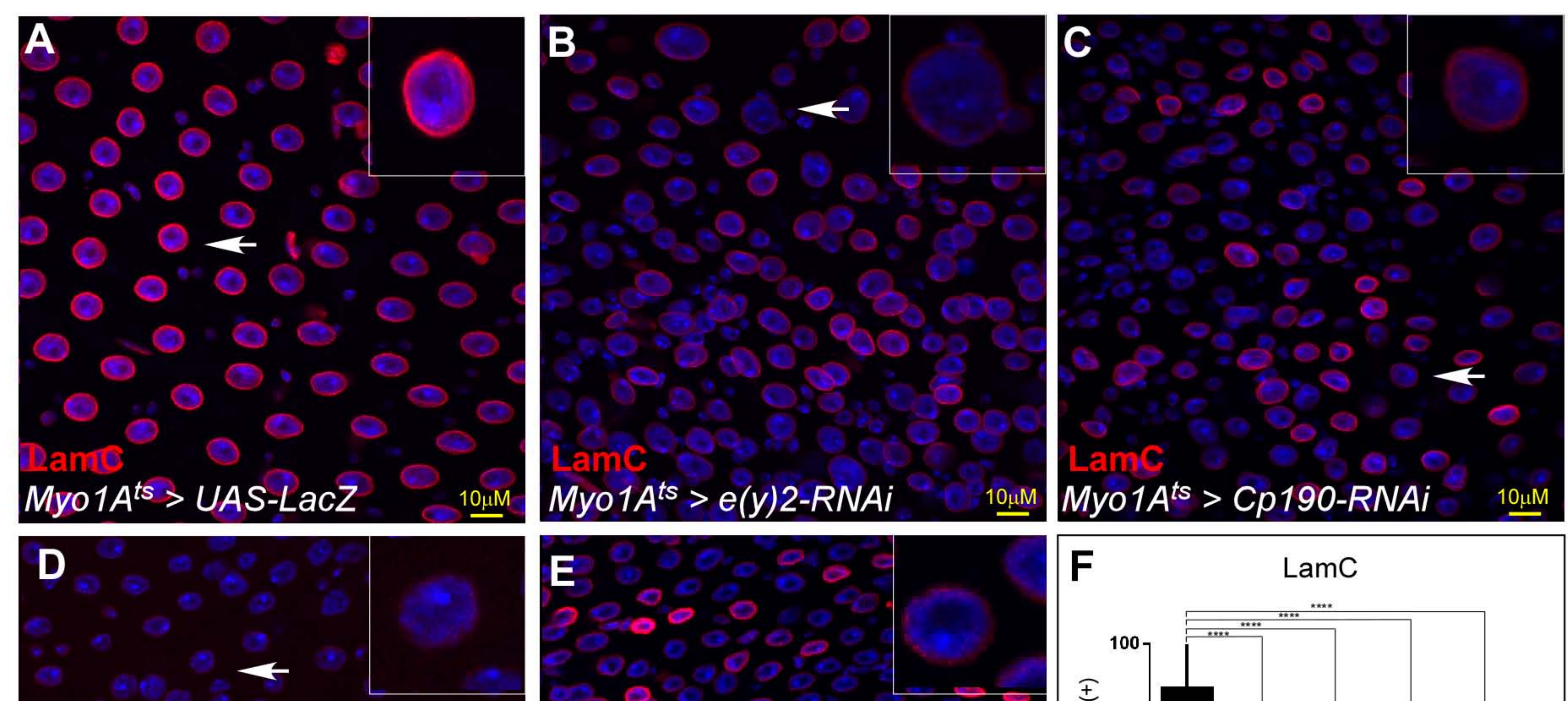

LamC

Myo1 $A^{\text {ts }}>$ Nup98-96-RNAi
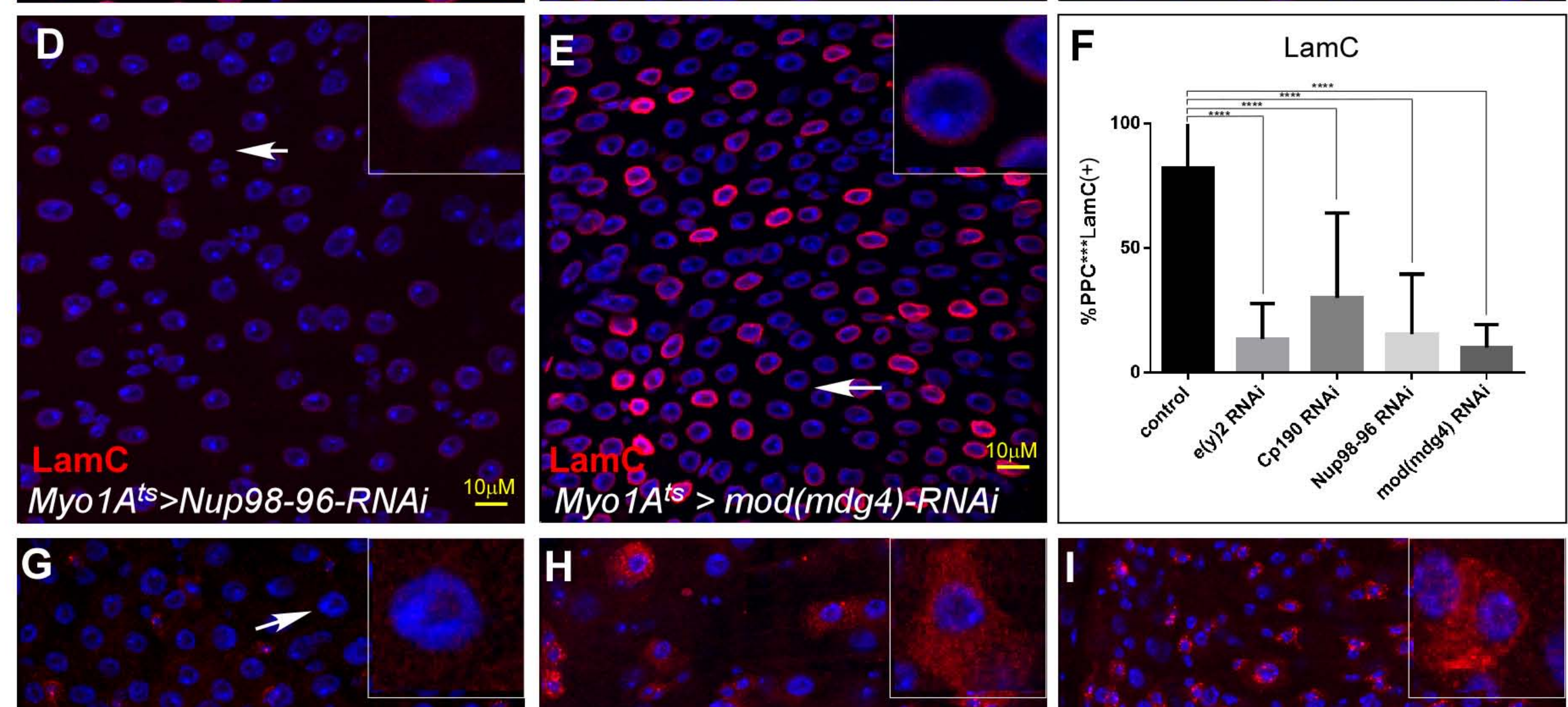

Delta

Myo1 $A^{\text {ts }}>$ UAS-LacZ
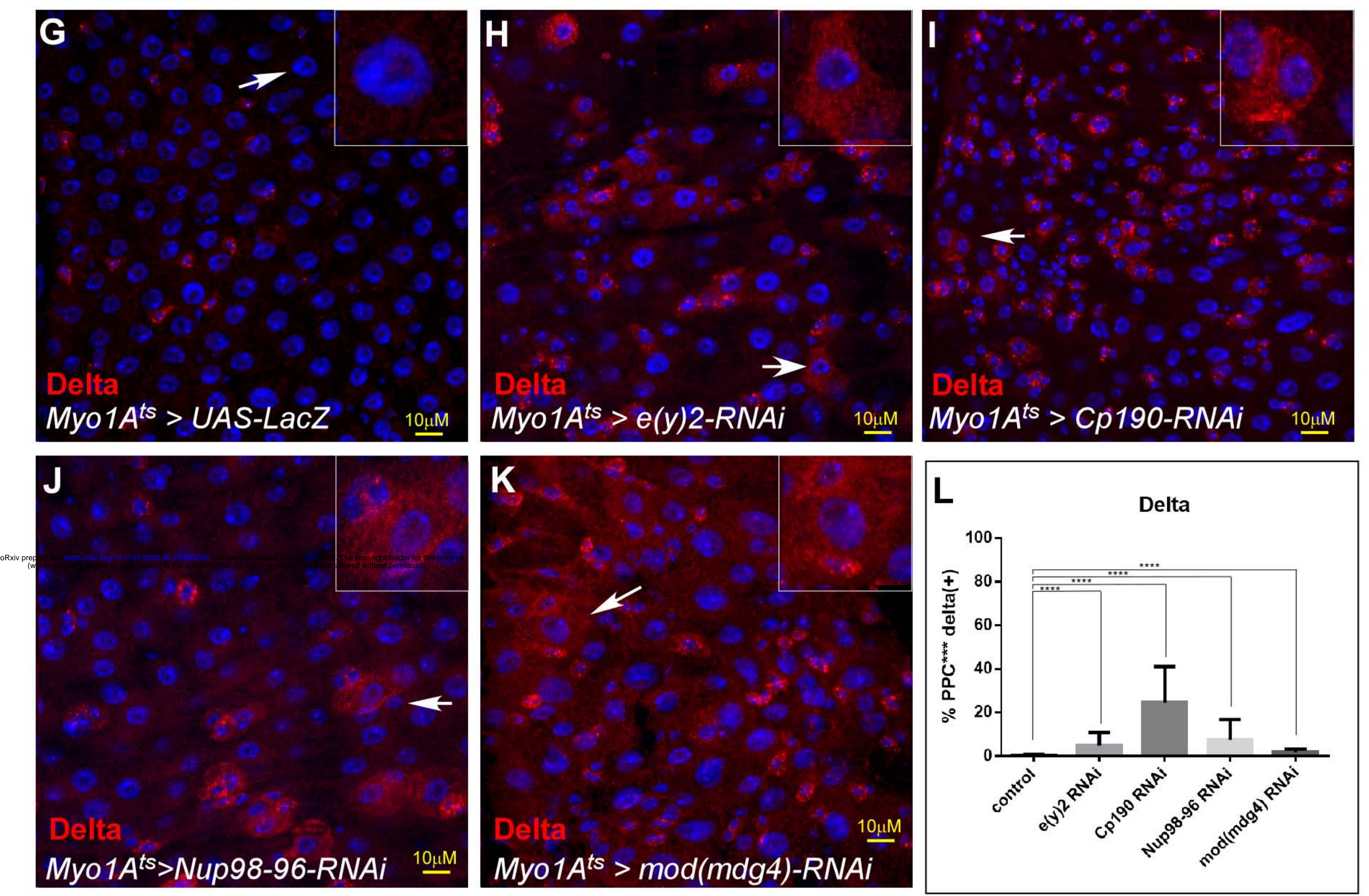
Figure 5 Fig. supp. 1; Erez et al.

A

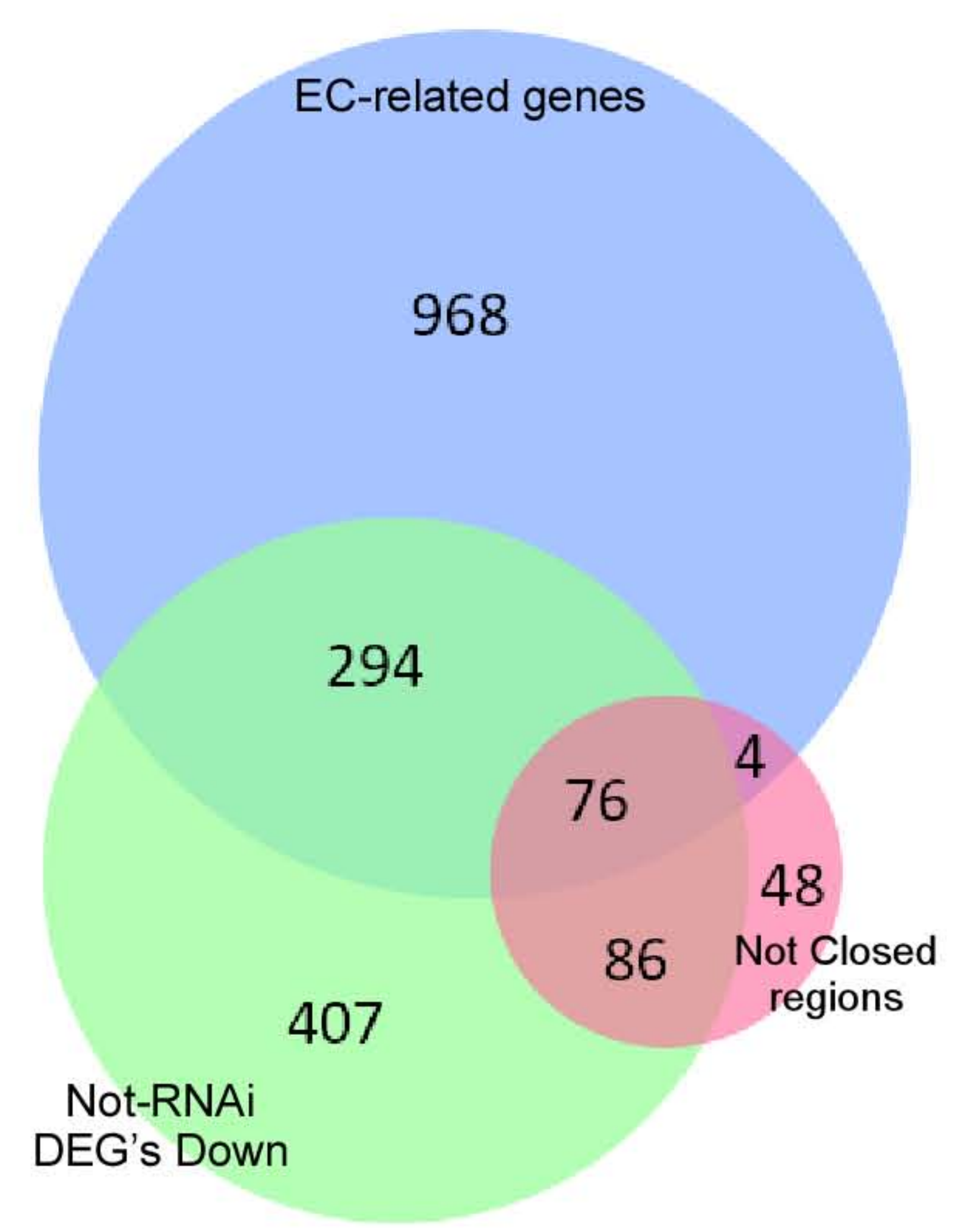

C

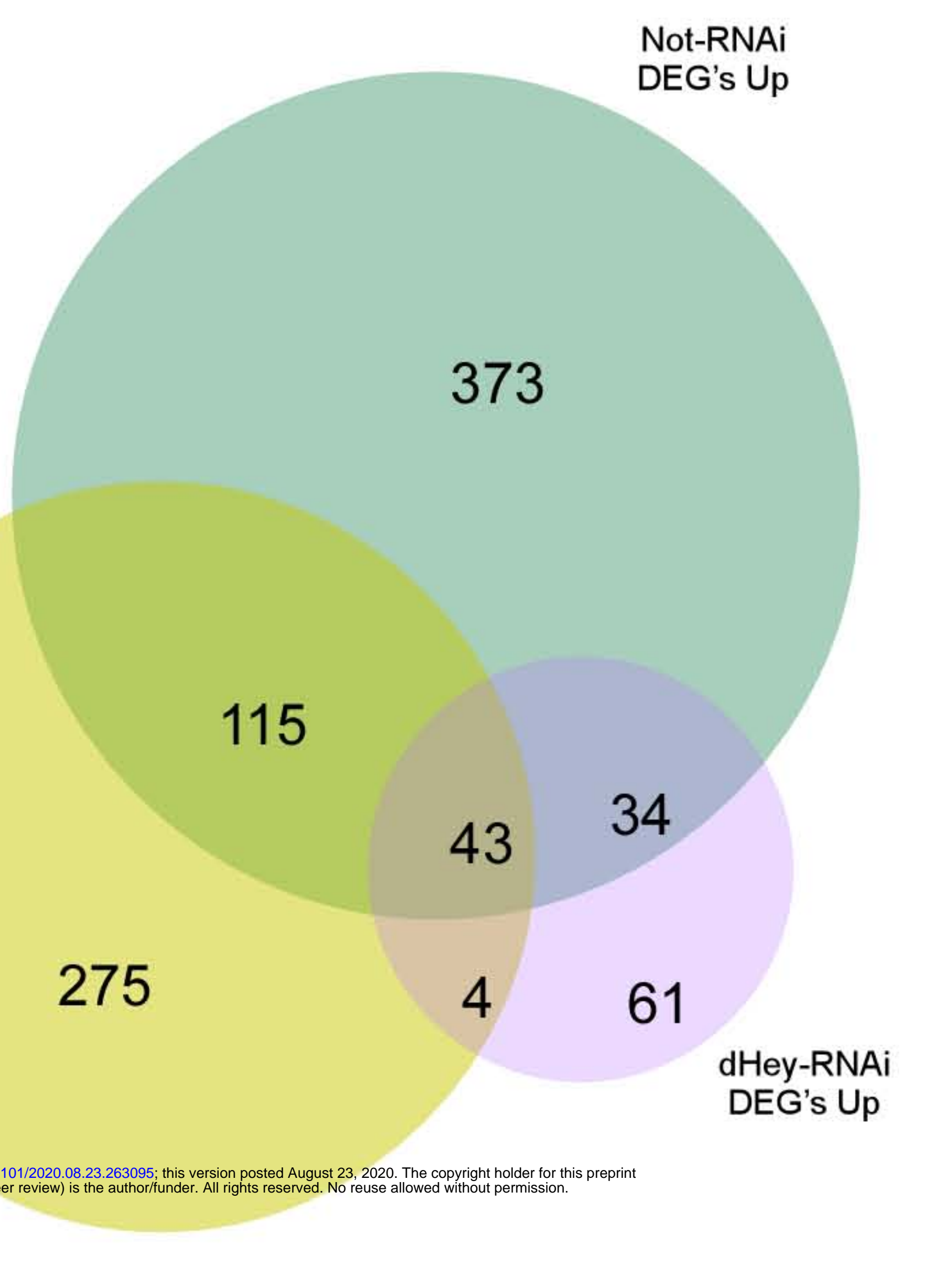

B

$$
\begin{aligned}
& \text { LamDmo } \\
& \text { OE DEG's }
\end{aligned}
$$

184

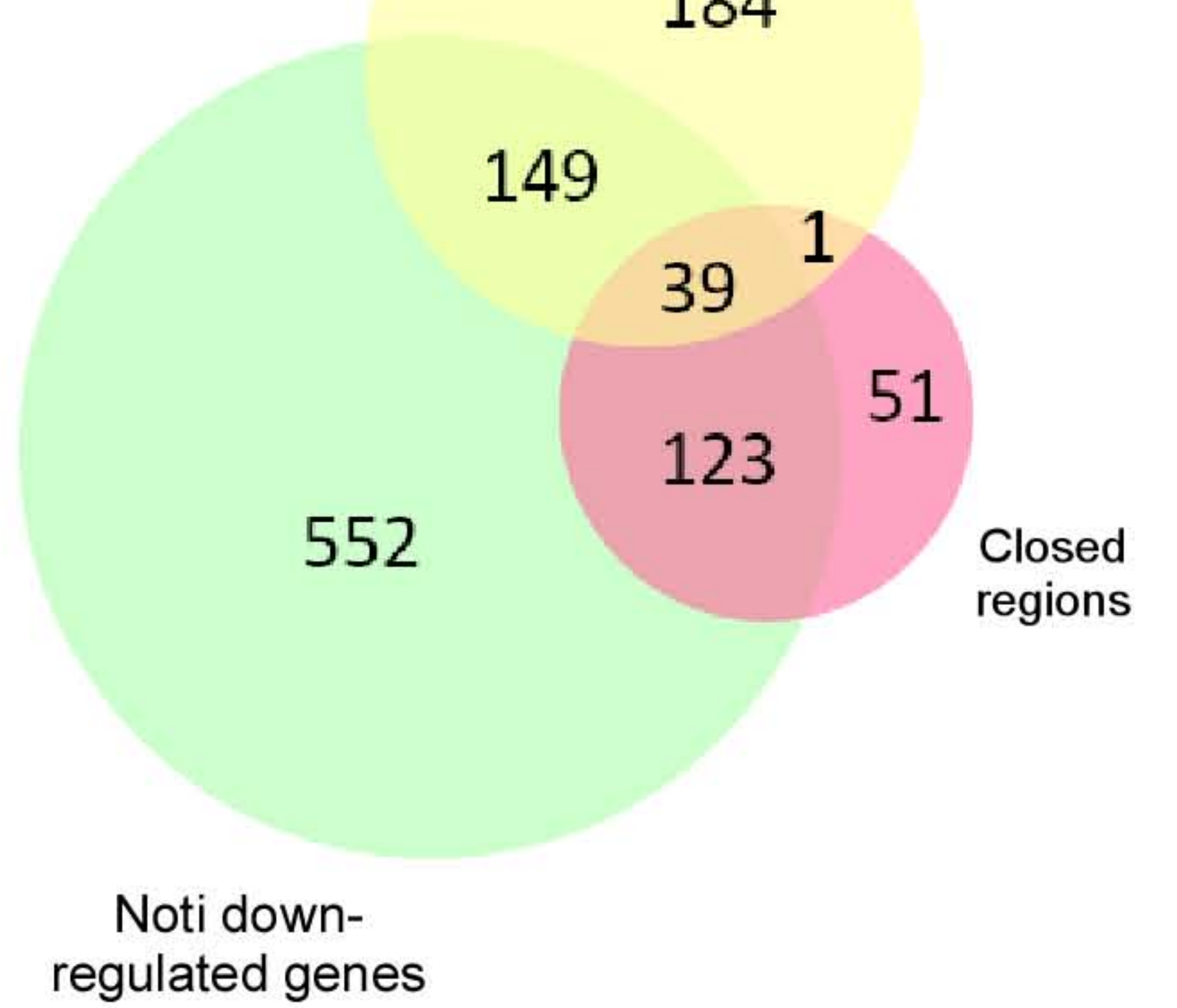

regulated genes
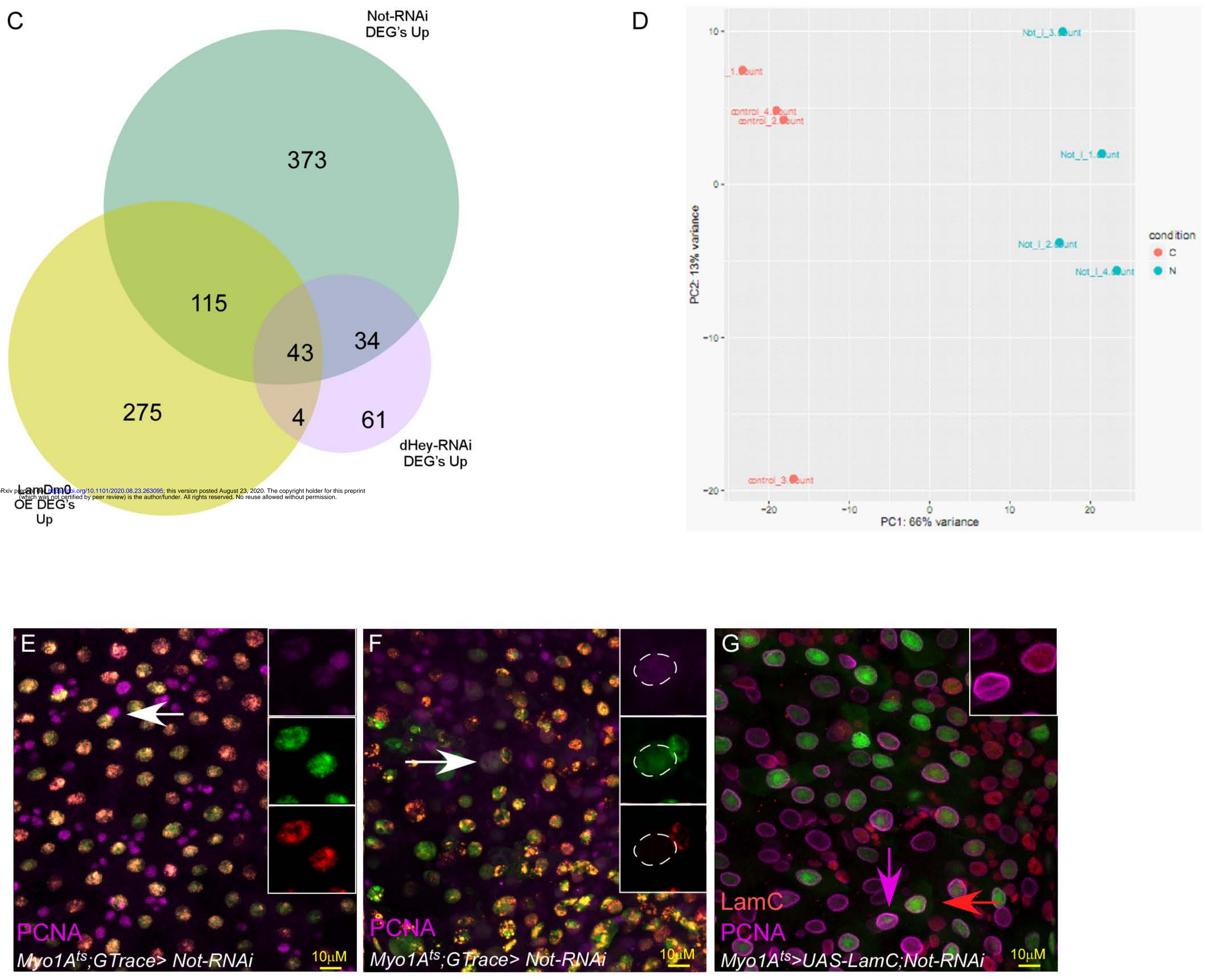
Figure 5 Fig supp 2: Erez et.al.

A.

Cell fate com mitment

Tube development

Tube formation

pattern specification process

Regulation of organ

morphogenesis

Positive regulation of nitrogen compound metabolic process Regulation of cellular

Cluster 2 component biogenesis Cation homeostasis Maintenance of location

Response to ecdysone Cytoskeleton organization Drug catabolic process

Regulation of multi-organism process

Dorsal/ventral pattern formation Cellular com ponent assembly involved in morphogenesis Arginine and proline metabolism Cofactor metabolic process Metal ion homeostasis

Wing disc pattern formation Behavior

Phosphorylation

Locomotor rhythm

Cluster 3 mRNA catabolic process Monosaccharide metabolic process

Response to endogenous stimulus

Germarium-derived egg

chamber formation

Response to alcohol

Cluster 9

Response to starvat

Syncytium formation by

plasma membrane fusion

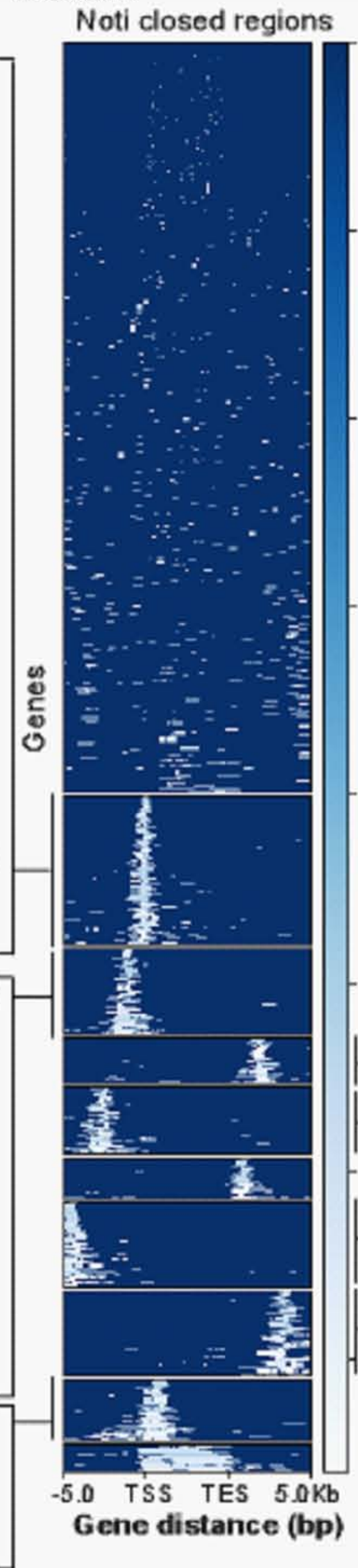

C. Genes in Open regions

\begin{tabular}{|l|l|}
\hline FlyBase ID & SYMBOL \\
\hline FBgn0267472 & asRNA:CR45822 \\
FBgn0267160 & asRNA:CR45600 \\
FBgn0038290 & CG6912 \\
FBgn0031602 & CG15431 \\
FBgn0038291 & CG3984 \\
FBgn0051344 & CG31344 \\
FBgn0265671 & InCRNA:CR44478 \\
FBgn0038299 & Spn88Eb \\
FBgn0264911 & CG44102 \\
FBgn0262357 & CG43055 \\
FBgn0266818 & IncRNA:CR45280 \\
FBgn0041184 & Socs36E \\
FBgn0038301 & CG6654 \\
FBgn0267485 & asRNA:CR45835 \\
FBg0033153 & Gadd45 \\
FBgn0042701 & CR12628 \\
\hline
\end{tabular}

Cellular response to chemical stimulus

Response to organic substance Cluster 4

Olfactory behavior

Glutathione metabolic process Cluster 5

Vesicle-mediated process

Cofactor metabolic process

Maintenance of location

Olfactory behavior

Coenzyme metabolic process

Mitochondrion organization

Positive regulation of multicellular

organismal process

Response to organic cyclic compound Cluster 8

B.

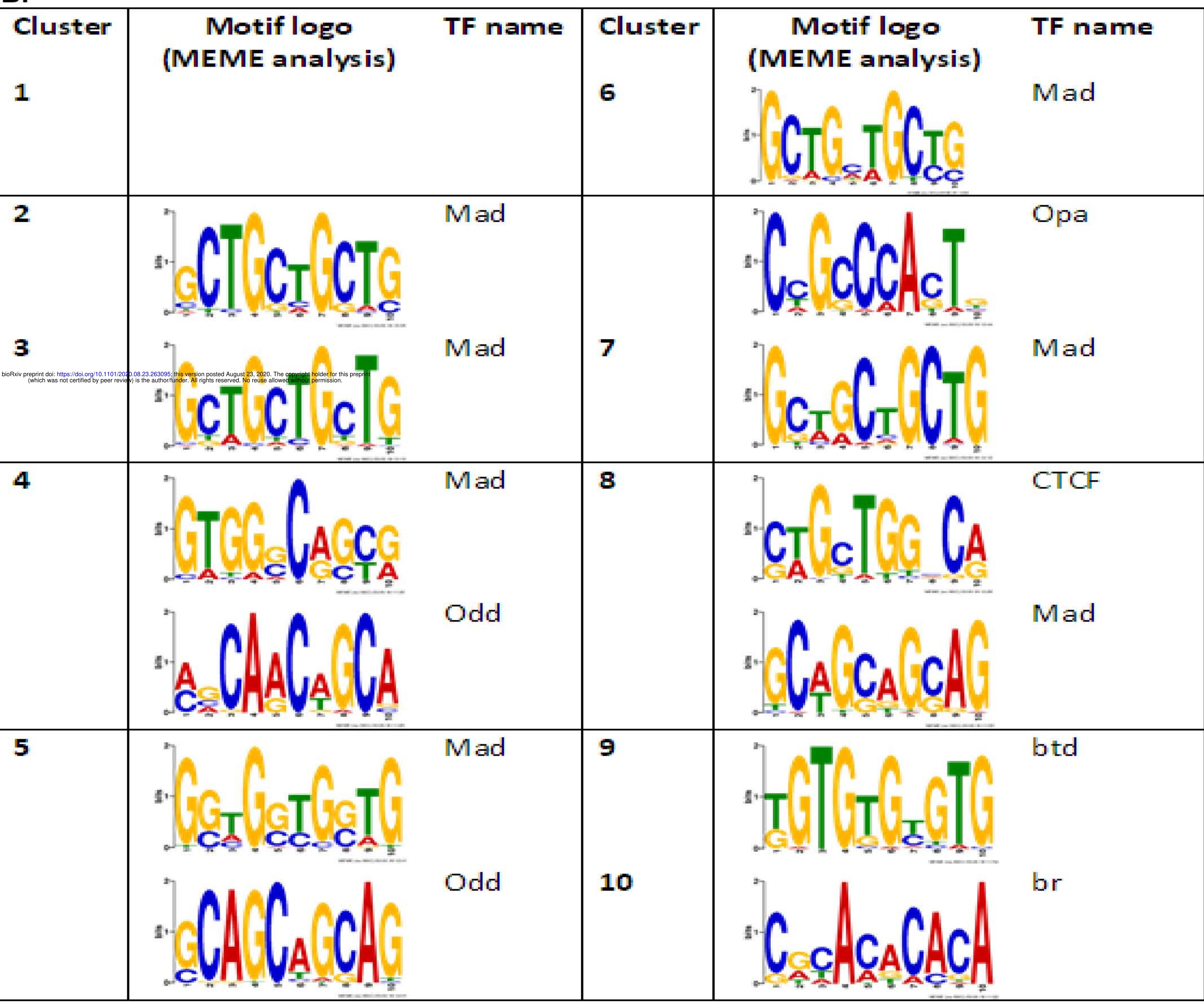


Fig 6 - Erez et al




Figure 6 Fig. Supp 1 Erez et al.


Nop60B

Myo1 $A^{\text {ts }}$;Dicer>mod(mdg4)-RNAi

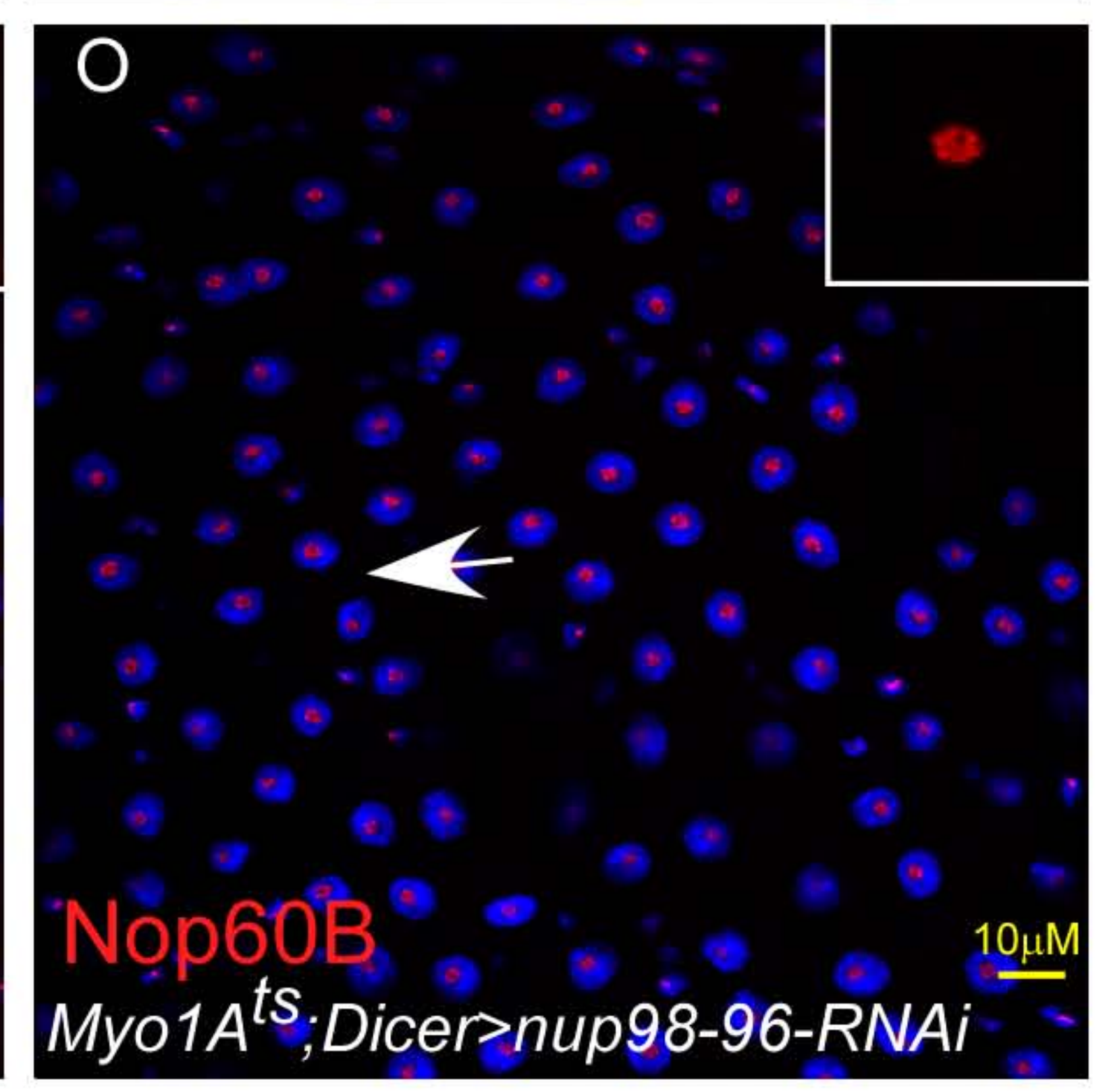

Nop60B

Myo1A ${ }^{\text {ts }}$;Dicer>e(y)2-RNAi 10um
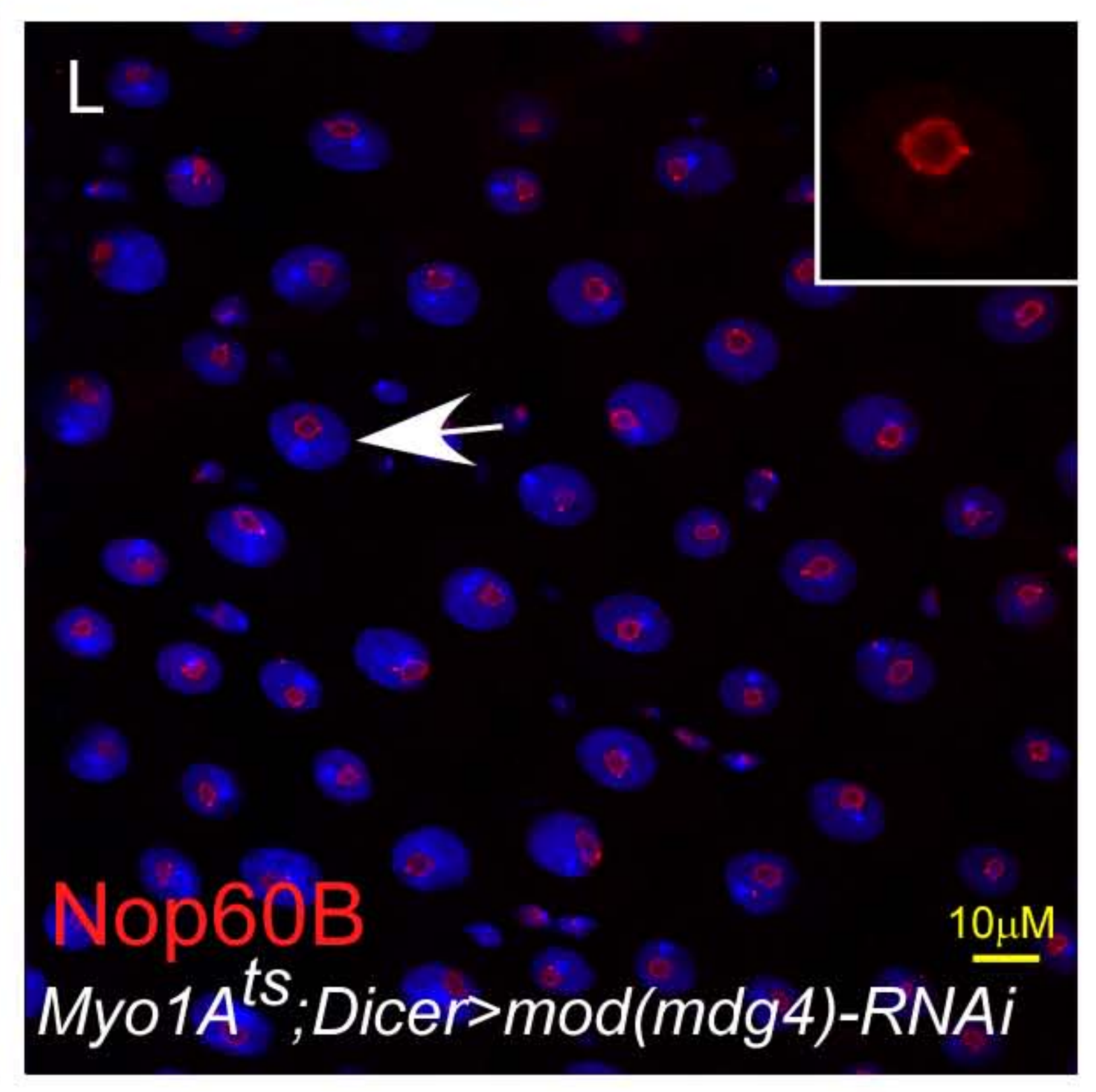
Fig 7 - Erez et al




Fig 8 - Erez et.al.




Figure 8 Fig. supp. 1: Erez et al




Fig 9 - Erez et al

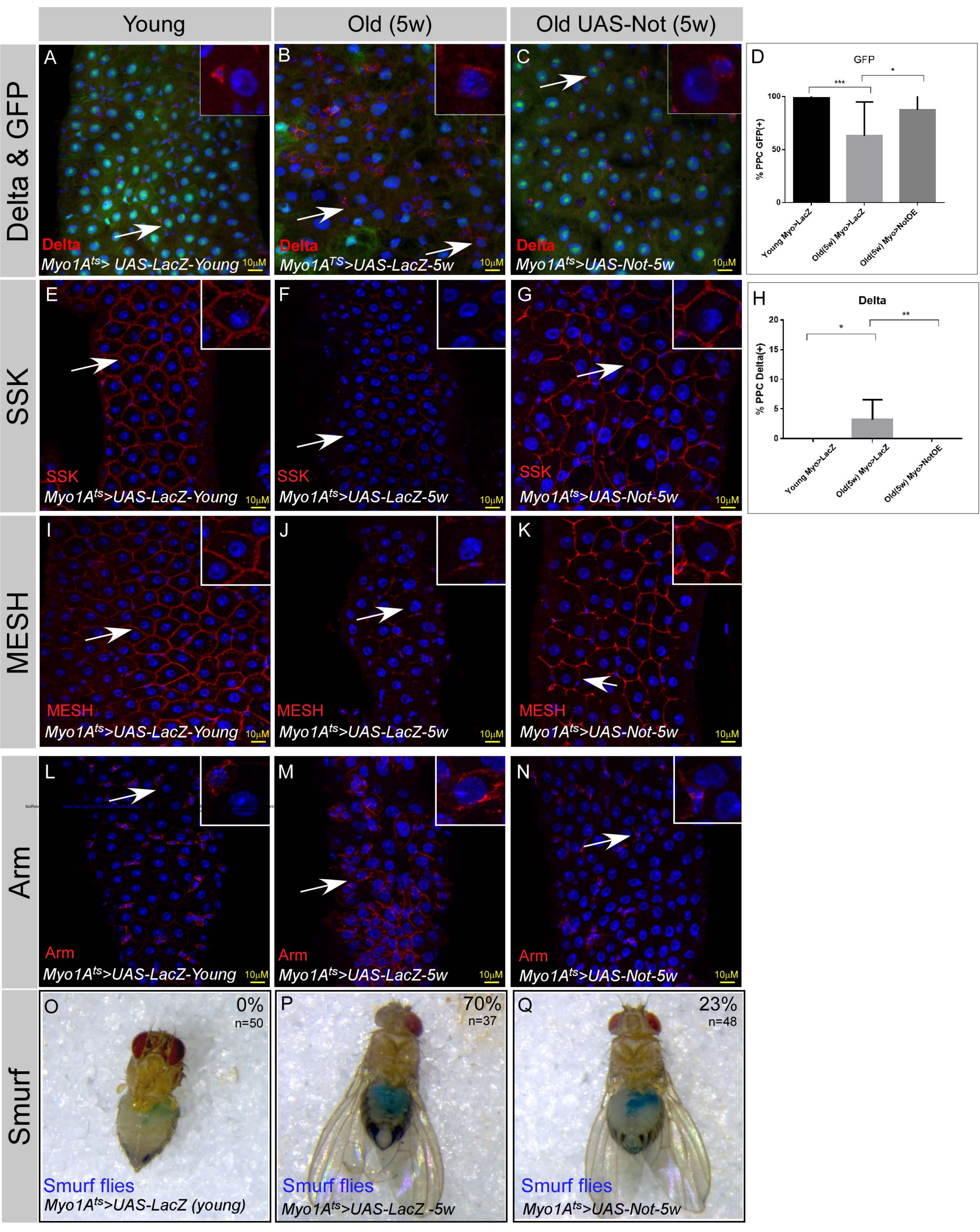

